\title{
Coated electrospun alginate-containing fibers as novel delivery systems for regenerative purposes
}

This article was published in the following Dove Press journal:

International Journal of Nanomedicine

\author{
Barbara Vigani' \\ Silvia Rossi' \\ Giuseppina Sandri' \\ Maria Cristina Bonferoni' \\ Giulia Milanesi' \\ Giovanna Bruni² \\ Franca Ferrari' \\ 'Department of Drug Sciences, \\ University of Pavia, Pavia, Italy; \\ ${ }^{2}$ Department of Chemistry, University \\ of Pavia, Pavia, Italy
}

\begin{abstract}
Aim: The aim of the present work was to develop biodegradable alginate (ALG)-containing fibrous membranes intended for tissue repair, acting as both drug delivery systems and cell growth guidance.

Methods: Membranes were prepared by electrospinning. Since ALG can be electrospun only when blended with other spinnable polymers, dextran (DEX) and polyethylene oxide (PEO) were investigated as process adjuvants. ALG/DEX mixtures, characterized by different rheological and conductivity properties, were prepared in phosphate buffer or deionized water; surfactants were added to modulate polymer solution surface tension. The Design of Experiments (DoE) approach (full factorial design) was used to investigate the role of polymer solution features (rheological properties, surface tension, and conductivity) on electrospun fiber morphology. A high viscosity at $1,000 \mathrm{~s}^{-1}(1.3-1.9$ Pa.s $)$ or a high pseudoplasticity index $(\geq 1.7)$, combined with a low surface tension $(30-32 \mathrm{mN} / \mathrm{m})$ and a low conductivity $(800-1,000 \mu \mathrm{S} / \mathrm{cm})$, was responsible for the production of ALG/DEX homogeneous fibers. Such ranges were successfully employed for the preparation of ALG-containing fibers, using PEO, instead of DEX, as process adjuvant. ALG/DEX and ALG/ PEO fibers were subsequently subjected to cross-linking/coating processes to make them slowly biodegradable in aqueous medium. In particular, ALG/PEO fibers were cross-linked and coated with $\mathrm{CaCl}_{2} /$ chitosan solutions in water/ethanol mixtures. Due to DEX high content, ALG/DEX fibers were soaked in a polylactide-co-glycolide (PLGA) solution in ethyl acetate.

Results: Both cross-linking and coating processes made fibers insoluble in physiological medium and produced an increase in their mechanical resistance, assessed by means of a tensile test. PLGA-coated ALG/DEX and chitosan-coated ALG/PEO fibers were biocompatible and able to support fibroblast adhesion.
\end{abstract}

Conclusion: The DoE approach allowed to draw up guidelines useful for the preparation of homogeneous fibers, starting from mixtures of ALG and non-ionic polymers. Such fibers, upon coating, resulted to be good cell substrates, allowing cell adhesion and growth.

Keywords: DoE approach, ALG-based fibers, rheological properties, conductivity, surface tension, chitosan, PLGA

\section{Introduction}

In the last decades, electrospun fibers, having diameters in the nano- to micro-scale, have gained an increasing interest for a broad spectrum of biomedical applications, such as tissue engineering scaffolds, drug delivery systems, and wound dressings. ${ }^{1-5}$ The attractiveness of such fibrous membranes lies in their ability to mimic the threedimensional structure of the native extracellular matrix due to their peculiar morphological features, which can be tailored depending on the intended application. ${ }^{6}$ In particular, high surface-to-volume ratio and high porosity, which are peculiar features of electrospun submicron fibers, represent pivotal features for 1) cell adhesion and proliferation, 2) drug loading, and 3) oxygen exchange that is required to
Department of Drug Sciences, University of Pavia, Viale Taramelli 12, 27I00 Pavia, Italy

Tel +39 382987357

Fax +3938242 2975

Email silvia.rossi@unipv.it
6531

Dovepress f in 0

http://dx.doi.org// 0.2147 IIN.S175069 
enhance wound healing when fibers are used as dressings. ${ }^{7-9}$ In addition, simplicity, cost-effectiveness, and versatility of the electrospinning technique make fibers even more appealing systems. ${ }^{10}$

To date, more than 200 polymers, both natural and synthetic, have been successfully electrospun. ${ }^{10,11}$ The choice of polymer represents a crucial step in the development of fibrous systems via electrospinning: molecular weight and chain length of polymer may affect the morphology and, thus, the degradation rate, swelling behavior, and mechanical properties of electrospun fibers. ${ }^{12}$

Alginate (ALG) is a natural anionic polysaccharide, derived from marine brown algae. It is widely used in the pharmaceutical field due to its water solubility, biocompatibility, low immunogenicity, and relative low cost. ${ }^{13}$ However, the production of ALG submicron fibers by electrospinning is still challenging; many research groups have attempted to electrospin aqueous solutions of pure ALG without success. ${ }^{14-19}$ ALG solutions, even at low concentrations $(2 \% \mathrm{w} / \mathrm{v}$ in deionized water), are too viscous to flow through the needle tip of the electrospinning apparatus. Meanwhile, ALG concentrations $<2 \% \mathrm{w} / \mathrm{v}$ do not guarantee adequate polymer chain entanglements needed to obtain a continuous jet that, in turn, is necessary for the production of homogenous fibers. ${ }^{14}$ Moreover, ALG is a polyelectrolyte, and therefore, it is characterized by a high conductivity; the strong repulsive forces between the polyanionic ALG chains represent another important factor, which can impair the electrospinnability of such biopolymer. ${ }^{15,18}$

In the last decades, some research groups have succeeded in the preparation of electrospun ALG-based fibers by blending ALG with a second polymer, such as polyethylene oxide (PEO) or polyvinyl alcohol, or a co-solvent, such as glycerol. The addition of such spinnable enhancers may decrease the viscosity of ALG solution, reduce the repulsive charges between ALG molecules, and improve chain flexibility. ${ }^{19-21}$

The aim of the present work was to develop biodegradable ALG-containing fibrous platforms intended for tissue repair, acting as both drug delivery systems and cell growth guidance. Such fibrous platforms were prepared by electrospinning.

ALG was chosen as key component of the fiber core for its capability to form complexes with cationic drugs and to produce hydrogels in the presence of bivalent cations such as $\mathrm{Ca}^{2+}$; these properties are functional for a controlled drug delivery. ${ }^{20}$ Since ALG can be electrospun when blended with other spinnable polymers, dextran (DEX) and PEO, having different nature and rheological properties, have been investigated as process adjuvants.

DEX is a bacterial-derived polysaccharide, biocompatible, biodegradable, characterized by a high water solubility and represents a novel polymer to be investigated for electrospun fiber production. ${ }^{4,21,22}$ On the contrary, PEO is a synthetic nonionic polymer, widely used for electrospinning. ${ }^{23}$ PEOs with different molecular weights (from 200,000 to 7,000,000 Da) have gained FDA approval only for oral administration, but in several papers, this polymer is used for the production of scaffolds intended for biomedical applications. ${ }^{24}$

It is known that the morphology of electrospun fibers is strictly related to polymeric solution properties, as well as to process (applied voltage, spinneret-collector distance, and flow rate) and environmental (temperature and relative humidity) parameters. ${ }^{10,25}$ So far, little work has been done to understand the relation between solution properties, such as rheology, surface tension, and conductivity, and the morphology of the resultant fibers. For this reason, in the present paper, the Design of Experiments (DoE) approach (full factorial design) was used to investigate the role of each polymer solution property on the preparation of homogeneous ALG/DEX fibers. In particular, rheological parameters such as viscosity at high shear and pseudoplasticity index, surface tension, and conductivity were determined. The aim was to identify the optimal values of each parameter to be used as guidelines for the production of ALG-containing fibers, independently of the polymer adjuvant used. In order to verify the predictive power of such guidelines, they were employed for the preparation of ALG-containing fibers, using PEO, instead of DEX, as polymer adjuvant of the electrospinning process. ALG/PEO mixtures with pseudoplasticity index, surface tension, and conductivity values in the ranges previously determined were electrospun, and the morphology of the products obtained was assessed.

In order to slowly obtain erodible fibers when kept in contact with biological fluids, the developed ALG/DEX and ALG/PEO fibers were coated. In particular, ALG/PEO fibers, previously cross-linked with calcium ions, were coated with chitosan (CS), a cationic polysaccharide, known for its antimicrobial and antioxidant properties, ${ }^{26}$ whereas ALG/DEX fibers were coated with polylactide co-glycolide (PLGA), a well-known biodegradable polymer. Mechanical properties of the coated and uncoated fibers were investigated by means of a tensile apparatus.

Fibrous platform cytocompatibility and capability to act as cell support were assessed in vitro using human fibroblasts to mimic dermal environment. 


\section{Materials and methods Materials}

Alginic acid sodium salt, from brown algae (ALG, medium viscosity, Brookfield viscosity $\geq 2,000 \mathrm{cps}$ for $2 \%$ solution in water at $25^{\circ} \mathrm{C}, \mathrm{G} / \mathrm{M}$ ratio equal to $70 / 30$; Sigma-Aldrich Co., St Louis, MO, USA), DEX 500 (Pharmacosmos, Holbaek, Denmark), PEO (Mw =4,000 kDa; Colorcon, Dartford, UK), Kolliphor P407 poloxamer (P407; BASF SE, Ludwigshafen, Germany), and Triton X-100 (TX100; Fluka BioChemika, Buchs, Switzerland) were used for the preparation of ALG/ DEX and ALG/PEO solutions. Phosphate buffer ( $\mathrm{pH}$ 5.5), prepared according to European Pharmacopeia, was used as solvent for the preparation of ALG/DEX solutions. Resomer $\mathrm{R} 503 \mathrm{H}$, poly(D,L-lactide-co-glycolide) acid terminated (PLGA, lactide:glycolide 50:50, Mw =24,000-38,000; Sigma-Aldrich Co.) and ethyl acetate (Carlo Erba, Milan, Italy) were used for ALG/DEX fiber coating.

Anhydrous calcium chloride $\left(\mathrm{CaCl}_{2}\right)$ and absolute ethanol $(\mathrm{EtOH})$ were purchased from Carlo Erba and used for ALG/PEO fiber cross-linking. ChitoClear ${ }^{\circledR}$ CS (degree of deacetylation 98\%; Primex, Siglufjordur, Iceland) and acetate buffers at $\mathrm{pH} 4.5$ and 5.1, prepared according to European Pharmacopeia, were used for the coating of ALG/ PEO cross-linked fibers.

For experiments with fibroblasts, the materials hereafter reported were used. Dimethyl sulfoxide (DMSO), Dulbecco's PBS, MTT, antibiotic antimycotic solution (100×; stabilized with 10,000 units penicillin, $10 \mathrm{mg}$ streptomycin, and $25 \mu \mathrm{g}$ amphotericin B per $\mathrm{mL}$ ), trypan blue solution, and trypsinEDTA solution were purchased from Sigma-Aldrich Co. DMEM was purchased from Corning Incorporated (Corning, NY, USA) and inactivated fetal bovine serum from Biowest (Nuaillé, France). Glutaraldehyde solution (Sigma-Aldrich Co.) was used to fix the cells cultured on ALG/DEX and ALG/PEO fibers prior to scanning electron microscopy (SEM) analysis.

\section{Assessment of the rheological interaction between ALG and DEX}

In order to assess the occurrence of an interaction between ALG and DEX, mixtures containing 30\% w/w DEX and increasing concentrations of ALG $(0.5 \%, 1 \%$, and $1.5 \%$ $\mathrm{w} / \mathrm{w}$ ) were subjected to viscoelastic measurements by means of a rotational rheometer (MCR 102; Anton Paar, Turin, Italy) equipped with a cone plate combination (CP50-1, diameter $=50 \mathrm{~mm}$; angle $=1^{\circ}$ ) as measuring system. Solutions of the individual polymers, having same concentrations as in the mixtures, were subjected to the same characterization. All measurements were carried out at $25^{\circ} \mathrm{C}$ after a rest time of 1 minute. Stress sweep and oscillation tests were performed.

In the stress sweep, increasing stresses were applied at a constant frequency $(1 \mathrm{~Hz})$, and the elastic response of the sample, expressed as storage modulus $\mathrm{G}^{\prime}$, was measured. Such a test allows to identify the "linear viscoelastic region." In the oscillation test, a shear stress, chosen in the linear viscoelastic region previously determined, was applied at increasing frequencies $(1-20 \mathrm{~Hz})$; the elastic response of the sample was measured as a function of frequency. The interaction parameter $\Delta \mathrm{G}^{\prime}$ was calculated, at $10 \mathrm{~Hz}$ frequency, according to the following equation: ${ }^{27}$

$$
\Delta \mathrm{G}^{\prime}=\mathrm{G}_{\mathrm{mix}}^{\prime}-\left(\mathrm{G}_{\mathrm{ALG}}^{\prime}+\mathrm{G}_{\mathrm{DEX}}^{\prime}\right)
$$

where

$\mathrm{G}_{\text {mix }}^{\prime}=$ storage modulus $(\mathrm{Pa})$ of DEX $30 \% \mathrm{w} / \mathrm{w}-\mathrm{ALG}$ $(0.5 \%, 1 \%$, and $1.5 \% \mathrm{w} / \mathrm{w})$ mixture.

$\mathrm{G}^{\prime}{ }_{\mathrm{ALG}}+\mathrm{G}_{\mathrm{DEX}}^{\prime}=$ theoretical value $(\mathrm{Pa})$ calculated as the sum of $\mathrm{G}^{\prime}$ values of $30 \% \mathrm{w} / \mathrm{w}$ DEX solution and of ALG solution, having the same concentration as in the mixture.

\section{Preparation of ALG/DEX-based solutions (D)}

D1-D8 formulations were prepared in deionized water or pH 5.5 phosphate buffer (Table 1): DEX (10\%-30\% w/w) and ALG $(0.5 \%-1.5 \% \mathrm{w} / \mathrm{w})$ were blended. Phosphate buffer was used instead of water to increase polymer solution conductivity. P407 and TX100 were added to ALG/DEX blends, alone or in combination, in the concentration range $0.5 \%-2 \% \mathrm{w} / \mathrm{w}$ to modulate polymer solution surface tension. D formulations were prepared at room temperature and maintained under stirring overnight.

\section{Characterization of ALG/DEX-based solutions (D)}

All the D formulations were characterized in terms of rheological properties, surface tension, and conductivity.

Table I Composition of D formulations, expressed as \% $\mathrm{w} / \mathrm{w}$

\begin{tabular}{llllll}
\hline Formulation & DEX & ALG & P407 & TXI00 & Solvent \\
\hline DI & 20 & $\mathrm{I}$ & 0 & $\mathrm{I} .5$ & $\mathrm{H}_{2} \mathrm{O}$ \\
D2 & 25 & $\mathrm{I} .5$ & $\mathrm{I} .5$ & 0.5 & $\mathrm{H}_{2} \mathrm{O}$ \\
D3 & 20 & $\mathrm{I}$ & $\mathrm{I} .5$ & 0 & $\mathrm{H}_{2} \mathrm{O}$ \\
D4 & 20 & $\mathrm{I} .5$ & 2 & 0 & $\mathrm{H}_{2} \mathrm{O}$ \\
D5 & 10 & $\mathrm{I} .5$ & $\mathrm{I} .5$ & 0.5 & $\mathrm{H}_{2} \mathrm{O}$ \\
D6 & 25 & $\mathrm{I} .5$ & $\mathrm{I} .5$ & 0.5 & $\mathrm{Buffer}$ \\
D7 & 10 & $\mathrm{I} .5$ & $\mathrm{I} .5$ & 0 & $\mathrm{H}_{2} \mathrm{O}$ \\
D8 & 30 & 0.5 & 2 & 0 & Buffer \\
\hline
\end{tabular}

Abbreviations: ALG, alginate; D, ALG/DEX-based solutions; DEX, dextran. 


\section{Rheological analysis}

Rheological analyses were performed by means of a rotational rheometer (MCR 102). Solution viscosity $(\eta)$ was measured at $33^{\circ} \mathrm{C}$ and at increasing shear rates in the range $10-1,000 \mathrm{~s}^{-1}$. Three replicates were performed for each formulation.

The pseudoplastic behavior of each polymeric solution was investigated by calculating the pseudoplasticity index (n) according to the following equation (power law model or Ostwald de Weale model): ${ }^{28}$

$$
\left(\tau-\tau_{0}\right)=\eta^{\prime} D^{n}
$$

where $\tau$ is the shear stress, $\tau_{0}$ the yield stress, $\eta$ ' the viscosity coefficient, $\mathrm{D}$ the shear rate, and $\mathrm{n}$ the pseudoplasticity index. The more $\mathrm{n}$ differs from 1 , the more the formulation deviates from a Newtonian behavior.

The viscoelasticity of D solutions was assessed by oscillation measurements, effected at $33^{\circ} \mathrm{C}$ by means of a rotational rheometer (MCR 102). Three replicates were considered for each sample.

\section{Dynamic surface tension measurements}

Dynamic surface tension measurements were carried out by means of an automatic tensiometer (DyneMaster DY-300; Kyowa Interface Science Co. Ltd., Saitama, Japan) at $33^{\circ} \mathrm{C}$. The analyses were performed by recording a surface tension value every 3 seconds up to 300 seconds. Three replicates were considered for each solution.

\section{Conductivity measurements}

Conductivity measurements were carried out by means of Mettler Toledo ${ }^{\mathrm{TM}}$ FiveGo $^{\mathrm{TM}} \mathrm{F} 3$ conductivity meter apparatus (Thermo Fisher Scientific). Three replicates were effected for each solution.

\section{Preparation and morphological characterization of ALG/DEX fibers}

All the D fibers were prepared by using an electrospinning apparatus (STIKIT-40; Linari Engineering, Grosseto, Italy), equipped with a high-voltage power supply, a syringe pump, and a collector plate, covered by an aluminum foil. Spinneret-collector distance, applied voltage, and flow rate were investigated in order to identify the best electrospinning conditions for each formulation. Polymeric solutions were pumped through a needle with a length $=15 \mathrm{~mm}$ and a gauge $=21$; the electrospinning process was performed at atmospheric pressure, maintaining constant temperature and relative humidity ranges, $27^{\circ} \mathrm{C}-33^{\circ} \mathrm{C}$ and $20 \%-30 \%$,

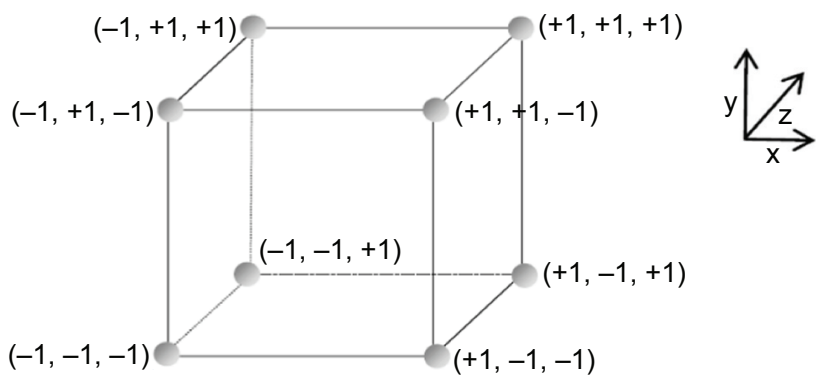

Figure I Factor space and experimenting points of a full factorial design.

respectively. Morphological evaluation was performed by means of a scanning electron microscope (EVO MA10; Carl Zeiss Meditec AG, Jena, Germany). Different magnifications $(2,000 \times, 5,000 \times$, and $10,000 \times)$ were considered for each fibrous membrane. Electrospun fiber dimensions were measured using the imaging analysis program ImageJ. Thirty fibers were considered for each sample.

\section{DoE (full factorial design)}

A full factorial design was employed to investigate, on a statistical basis, the role of each polymer solution parameter (viscosity, surface tension, and conductivity) on the production of homogeneous fibers without beads. Three factors corresponding to viscosity measured at high shear rate $\left(1,000 \mathrm{~s}^{-1}\right)$, surface tension, and conductivity were investigated at two different levels; three replicates were considered for each experiment (Figure 1). For each factor, two ranges of values, defined as low and high (indicated as -1 and +1 , respectively), were identified: $<1$ Pas and $>1.3$ Pas for viscosity, $<33 \mathrm{mN} / \mathrm{m}$ and $>38 \mathrm{mN} / \mathrm{m}$ for surface tension and $<1,000 \mu \mathrm{S} / \mathrm{cm}$ and $>1,400 \mu \mathrm{S} / \mathrm{cm}$ for conductivity. D solutions (D1-D8) were used for the full factorial design $2^{\mathrm{k}}(\mathrm{k}=3)$ (Table 2). Fiber morphology was considered as the response variable; a score from 0 to 10 was given: 10 (homogeneous fibers), 7.5 (irregular fibers), 5 (fibers with beads), 2.5 (fibers with numerous beads), and 0 (no fibers).

Table 2 Full factorial design experimental points

\begin{tabular}{llll}
\hline & Viscosity & Surface tension & Conductivity \\
\hline DI & $-I$ & $-I$ & $-I$ \\
D2 & $+I$ & $-I$ & $-I$ \\
D3 & $-I$ & $+I$ & $-I$ \\
D4 & $+I$ & $+I$ & $-I$ \\
D5 & $-I$ & $-I$ & $+I$ \\
D6 & $+I$ & $-I$ & $+I$ \\
D7 & -1 & $+I$ & $+I$ \\
D8 & $+I$ & $+I$ & $+I$ \\
\hline
\end{tabular}

Abbreviations: ALG, alginate; D, ALG/DEX-based solutions; DEX, dextran. 
A second full factorial design was performed to investigate the role of another factor, pseudoplasticity index, used in substitution of viscosity at high shear rate. Two ranges of values, defined as low and high (indicated as -1 and +1 , respectively), were identified for this parameter: $<1.7$ and $>1.7$.

\section{Preparation and morphological characterization of ALG/PEO fibers}

An ALG/PEO mixture, characterized by pseudoplasticity index, surface tension, and conductivity in the range identified by DoE approach as optimal for the production of homogenous fibers, was prepared in deionized water. In particular, 1.5\% w/w PEO 4,000 kDa was blended with $1 \% \mathrm{w} / \mathrm{w}$ ALG. P407 and TX100 were added to ALG/PEO mixture to achieve concentrations equal to $1.5 \%$ and $0.5 \% \mathrm{w} / \mathrm{w}$, respectively. The ALG/PEO mixture was prepared at room temperature and maintained under stirring overnight.

ALG/PEO fibers were prepared by using an electrospinning apparatus, as described in the "Preparation and morphological characterization of ALG/DEX fibers" section; different process parameters in particular spinneret-collector distance $(10-15 \mathrm{~cm})$, applied voltage $(20-25 \mathrm{kV})$, and flow rate $(0.397 \mathrm{~mL} / \mathrm{h})$ were investigated in order to identify the best electrospinning conditions. Morphological analyses were performed as previously described for ALG/DEX fibers (in the "Preparation and morphological characterization of ALG/DEX fibers" section).

\section{Coating of ALG/DEX and ALG/PEO fibers}

Fibers were subjected to ionic cross-linking and/or polymerbased coating. ALG/DEX fibers were soaked for 2 minutes in a solution of PLGA (R 503H) prepared in ethyl acetate and dried at room temperature. Two different concentrations of $\mathrm{R} 503 \mathrm{H}, 0.25 \% \mathrm{w} / \mathrm{v}$ and $0.5 \% \mathrm{w} / \mathrm{v}$, were considered.

A multistep protocol for the cross-linking and coating of ALG/PEO fibers was set up. Briefly, ALG/PEO fibers were subjected to a pre-cross-linking treatment with absolute ethanol for 5 minutes. Later, fibers were soaked in $2 \% \mathrm{w} / \mathrm{w}$ $\mathrm{CaCl}_{2}$ solution prepared in $80 \% \mathrm{v} / \mathrm{v}$ ethanol (cross-linked fibers) and, then, in $0.1 \% \mathrm{w} / \mathrm{w} \mathrm{CS} / 2 \% \mathrm{w} / \mathrm{w} \mathrm{CaCl}_{2}$ solution prepared in acetate buffer ( $\mathrm{pH}$ 4.5) (coated fibers). Crosslinked/coated fibers were washed with acetate buffer ( $\mathrm{pH}$ 5.0) and dried at room temperature. Morphological analyses on both coated ALG/DEX and ALG/PEO fibers were performed (as described in the "Preparation and morphological characterization of ALG/DEX fibers" section).

\section{Structural analyses of coated fibers}

Fourier transform infrared (FTIR) spectroscopy

FTIR spectra were obtained with a Nicolet FTIR iS10 spectrometer (Nicolet, Madison, WI, USA) equipped with attenuated total reflectance sampling accessory (Smart iTR with $\mathrm{ZnSe}$ plate) by co-adding 32 scans in the spectral region of 4,000-650 $\mathrm{cm}^{-1}$ with a resolution of $4 \mathrm{~cm}^{-1}$ resolution.

\section{Energy dispersive X-ray spectroscopy (EDS)}

EDS was performed using an FE-SEM (Tescan Mira3 XMU, Brno, Czech Republic), equipped with Apollo X Silicon Drift Detector.

\section{Assessment of mechanical properties of fiber}

The mechanical properties of fiber membranes were assessed by means of a TA.XT plus Texture Analyzer (Stable Micro Systems, Godalming, UK), equipped with 1 or $5 \mathrm{~kg}$ load cells. Before testing, fiber thickness was measured by means of Sicutool 3955 G-50 (Sicutool, Milan, Italy) apparatus.

Each fiber membrane was cut $(1 \times 3 \mathrm{~cm})$ and, then, clamped on A/TG tensile grips probe; an initial distance of $1 \mathrm{~cm}$ between the grips was set. The upper grip was raised at a constant speed of $5 \mathrm{~mm} / \mathrm{s}$ up to a distance of $5 \mathrm{~mm}$, corresponding to $50 \%$ elongation.

Maximum deformation force (Fmax), forces measured at different deformations (F10, F20, F30, F40, and F50 at $10 \%, 20 \%, 30 \%, 40 \%$, and $50 \%$ elongation, respectively), and deformation work (Wmax) were calculated for the optimized ALG/DEX and ALG/PEO fibers, as such, and after cross-linking/coating.

Moreover, the differential parameter $\Delta$ elongation at break $(\% \Delta \mathrm{E})$ was determined using the following equation: ${ }^{29}$

$\% \Delta \mathrm{E}=\frac{\text { Extension of length at rupture }- \text { Initial length }}{\text { Initial length }} * 100$

\section{In vitro cell culture experiments}

\section{Normal human dermal fibroblasts (NHDFs)}

NHDFs (Promocell GmbH, Heidelberg, Germany) from 6th to 12 th passage were used. Cells were cultured in complete medium (CM) consisting of DMEM supplemented with $1 \%$ $\mathrm{v} / \mathrm{v}$ antibiotic antimycotic solution and $10 \% \mathrm{v} / \mathrm{v}$ inactivated fetal calf bovine serum. After 24 hours UV irradiation, ALG/ DEX fibers, after PLGA-based coating with $0.25 \% \mathrm{w} / \mathrm{v} \mathrm{R}$ $503 \mathrm{H}$, and ALG/PEO fibers, after cross-linking and coating, were cut into pieces having a surface area of $\sim 0.32 \mathrm{~cm}^{2}$, placed in a 96-well plate and seeded with NHDF cells at a 
density of 35,000 cells/well. Thereafter, cells were maintained in incubator (Shellab ${ }^{\circledR}$ Sheldon ${ }^{\circledR}$ Manufacturing Inc., Cornelius, OR, USA) at $37^{\circ} \mathrm{C}$ with $95 \%$ air and $5 \% \mathrm{CO}_{2}$ atmosphere.

The effect of coated ALG/DEX and ALG/PEO fibers on cell viability was evaluated at 7 days by MTT assay, using $\mathrm{CM}$ as control. Briefly, after 7 days, CM was removed from each well and cells were rinsed with PBS; subsequently, $50 \mu \mathrm{L}$ of MTT $7.5 \mu \mathrm{M}$ in $100 \mu \mathrm{L}$ of DMEM without phenol red was added to each well and incubated for 3 hours $\left(37^{\circ} \mathrm{C}\right.$ and $5 \% \mathrm{CO}_{2}$ ). Finally, $100 \mu \mathrm{L}$ of DMSO, used as solubilization agent, was added to each well, in order to promote the complete dissolution of formazan crystals, obtained from MTT dye reduction by mitochondrial dehydrogenases of alive cells. The solution absorbance was measured by means of an iMark ${ }^{\circledR}$ Microplate reader (Bio-Rad Laboratories Inc., Hercules, CA, USA) at a wavelength of 570 and $690 \mathrm{~nm}$ (reference wavelength) after 60 seconds of mild shaking. Results were expressed as \% cell viability by normalizing the absorbance measured after contact with each sample with that measured for CM. Six replicates were performed for each sample.

A morphological study of fibroblasts on coated ALG/ DEX and ALG/PEO fibers was carried out after 7 days of culture. Cell distribution within both the ALG-based fibrous membranes was appreciated by SEM. After medium removal, the cell-fiber constructs were rinsed with PBS and, subsequently, fixed in $3 \% \mathrm{v} / \mathrm{v}$ glutaraldehyde solution. Samples were further rinsed with PBS, dehydrated with increasing concentration of ethanol $(25 \%, 50 \%, 75 \%$ and $100 \% \mathrm{v} / \mathrm{v})$ and dried at room temperature in a clean bench.

\section{Statistical analysis}

Whenever possible, experimental values of the various type of measures were subjected to statistical analysis, carried out by means of the statistical package Statgraphics 5.0 (Statistical Graphics Corporation, Rockville, MD, USA). In particular, one-way ANOVA - multiple range test and Student's $t$-test were used.

\section{Results}

It is known that PEO, when associated with ALG determines the formation of intermolecular hydrogen bonds, enhancing the entanglement degree of the polymeric blend, functional to the formation of fibers by electrospinning. ${ }^{18,30}$ To verify if an interaction occurs also between ALG and DEX, mixtures of DEX $30 \% \mathrm{w} / \mathrm{w}$ and ALG $(0.5 \%, 1 \%$, and $1.5 \%$ $\mathrm{w} / \mathrm{w})$ were subjected to viscoelastic measurements, and the results were compared to those obtained for the individual ALG and DEX solutions, having the same concentrations as in the mixtures. For all the frequencies considered, the mixtures are characterized by $\mathrm{G}^{\prime}$ values markedly higher than those of the individual polymer solutions (Supplementary materials). Recently, de Souza Ferreira et al proposed to calculate the rheological interaction parameter $\Delta \mathrm{G}^{\prime}$ as the difference between the $\mathrm{G}^{\prime}$ of the polymer blend and the theoretical value, which is the sum of $\mathrm{G}^{\prime}$ values of the individual solutions. ${ }^{27}$

In Figure 2, $\Delta \mathrm{G}^{\prime}$ values of $\mathrm{DEX} / \mathrm{ALG}$ mixtures are reported. An increase in the differential parameter is observed on increasing ALG concentration, indicating an increment of the interaction extent between the two polymers. Such an interaction is responsible for an improved entanglement that promotes electrospinning process.

A "full factorial design" (DoE approach) was employed to investigate, on a statistical basis, the role of each polymeric solution parameter (viscosity at high shear rate $\left[1,000 \mathrm{~s}^{-1}\right]$, surface tension, and conductivity) on the production of homogeneous fibers. Fiber morphology was considered as response variable: a score, from 0 to 10 , was given as reported in Table 3.

Figure 3 reports SEM micrographs that show the morphology of the products obtained from the ALG/DEX blends considered in the full factorial design. It can be observed that D5 solution does not produce fibers (Figure 3E), probably because DEX concentration is lower than Critical Entanglement Concentration (CEC), which is equal to $12.1 \% \mathrm{w} / \mathrm{w}$, in both distilled water and $\mathrm{pH} 5.5$ phosphate buffer (Supplementary materials). Also D7, characterized by the highest surface tension and conductivity values, does not produce fibers. The statistical analysis points out the effect of each factor and their interactions on the response variable. The Pareto

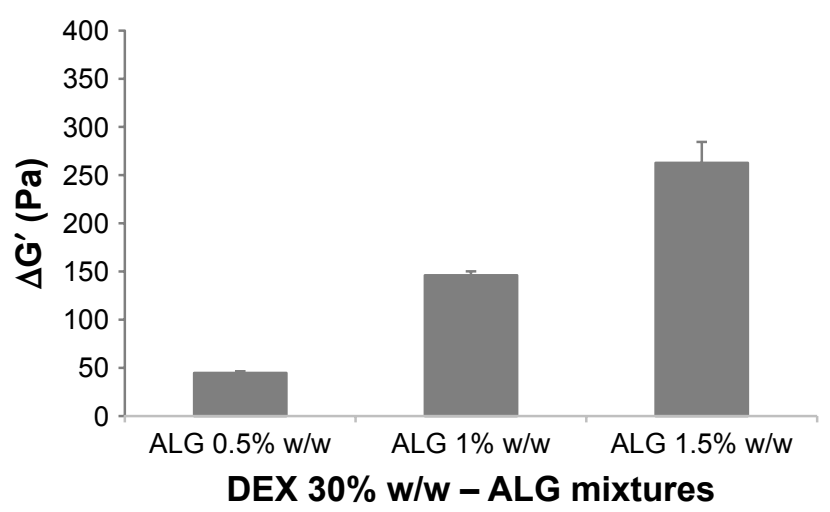

Figure $2 \Delta G^{\prime}$ values calculated for DEX $30 \%$ w/w/ALG mixtures containing increasing ALG concentrations $(0.5 \%, \mathrm{I} \%$, and $\mathrm{I} .5 \% \mathrm{w} / \mathrm{w}$ ) (mean values $\pm S D ; n=3$ ). Abbreviations: DEX, dextran; ALG, alginate. 
Table 3 Mean values ( $\left(S D ; n=3\right.$ ) of viscosity at $1,000 \mathrm{~s}^{-1}$, pseudoplasticity index, surface tension, and conductivity of each ALG/DEX formulation

\begin{tabular}{llllll}
\hline & $\begin{array}{l}\text { Viscosity at } \mathrm{I}, \mathbf{0 0 0} \mathbf{s}^{-1} \\
(\mathbf{P a} \cdot \mathbf{s})\end{array}$ & $\begin{array}{l}\text { Pseudoplasticity } \\
\text { index }\end{array}$ & $\begin{array}{l}\text { Surface tension } \\
(\mathbf{m N N} / \mathbf{m})\end{array}$ & $\begin{array}{l}\text { Conductivity } \\
(\mu \mathbf{S} / \mathbf{c m})\end{array}$ \\
\hline DI & $0.73 \pm 0.05$ & $1.618 \pm 0.029$ & $31.1 \pm 0.1$ & $613 \pm 22$ & $\begin{array}{l}\text { DoE score } \\
\text { D2 }\end{array}$ \\
\hline D3 & $1.88 \pm 0.05$ & $1.972 \pm 0.079$ & $31.0 \pm 0.7$ & $833.8 \pm 2$ & 10 \\
D4 & $1.01 \pm 0.02$ & $1.689 \pm 0.008$ & $41.2 \pm 1.1$ & $535 \pm 3$ & 2.5 \\
D5 & $1.32 \pm 0.14$ & $2.140 \pm 0.012$ & $38.3 \pm 1.8$ & $990 \pm 14$ & 5 \\
D6 & $0.40 \pm 0.01$ & $1.671 \pm 0.015$ & $33.0 \pm 0.1$ & $1,497 \pm 5$ & 0 \\
D7 & $1.68 \pm 0.04$ & $2.164 \pm 0.017$ & $31.3 \pm 1.0$ & $2,487 \pm 7$ & 7.5 \\
D8 & $0.44 \pm 0.04$ & $1.950 \pm 0.016$ & $39.0 \pm 0.2$ & $1,525 \pm 2$ & 0 \\
\hline
\end{tabular}

Note: The score attributed to each formulation according to the morphology of the corresponding electrospun fibers is also reported.

Abbreviations: D, ALG/DEX-based solutions; DEX, dextran; ALG, alginate; DoE, Design of Experiments.

chart, reported in Figure 4A highlights that both conductivity and surface tension determine a negative and statistically significant effect on fiber formation. This suggests that an increase in one of these parameters impairs the possibility to obtain homogeneous electrospun fibers. Viscosity at high shear rate shows a positive and statistically significant effect on the response variable. Both the interactions between surface tension and conductivity and between viscosity and conductivity positively influence the formation of fibers characterized by an optimal morphology (Figure 4A and B).
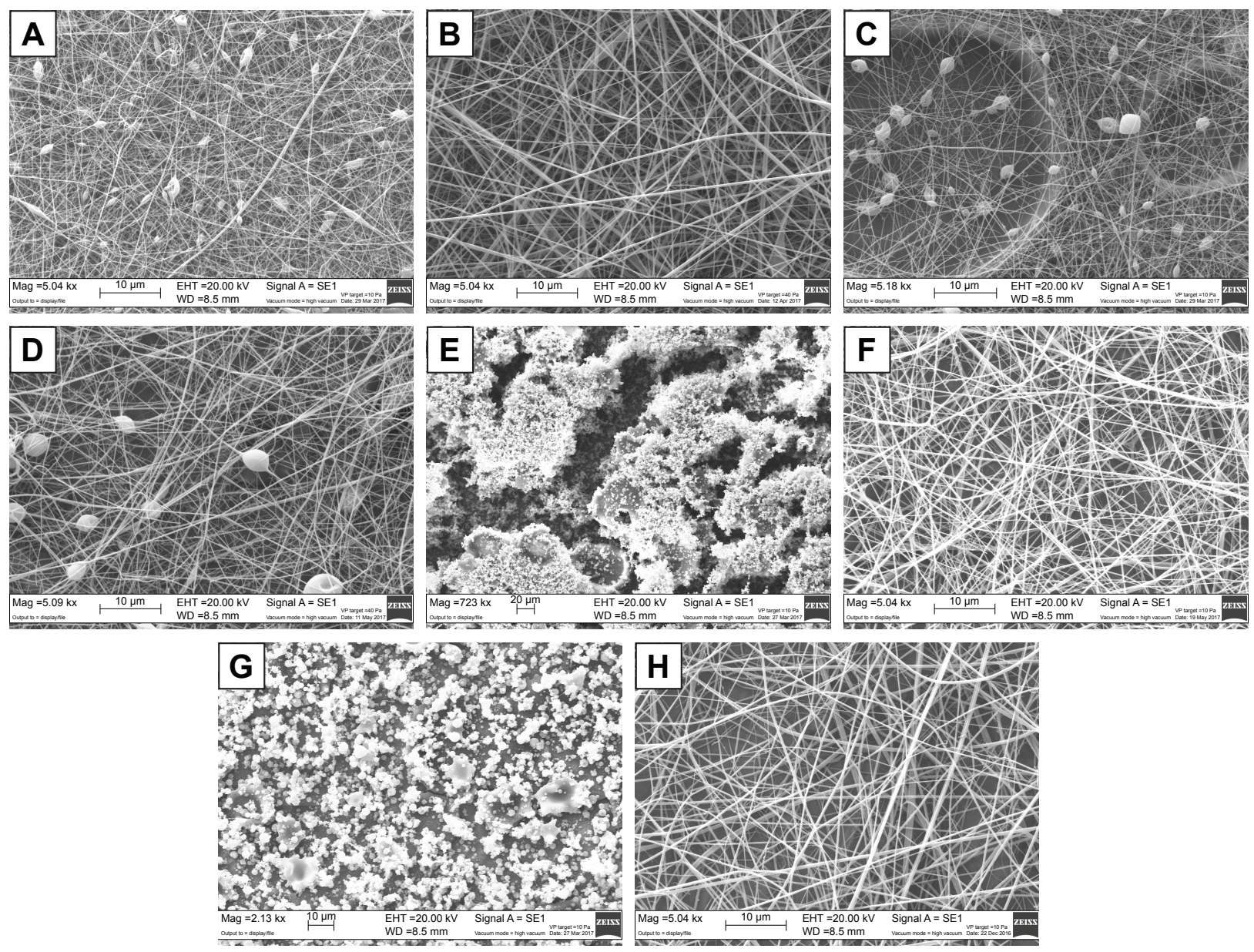

Figure 3 SEM micrographs of (A) DI, (B) D2, (C) D3, (D) D4, (E) D5, (F) D6; (G) D7, and (H) D8 products obtained from electrospinning. Abbreviations: ALG, alginate; D, ALG/DEX-based solutions; DEX, dextran; SEM, scanning electron microscopy. 
A

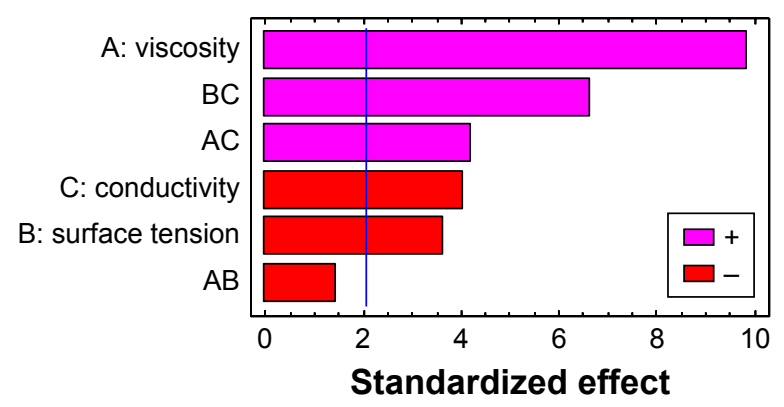

C

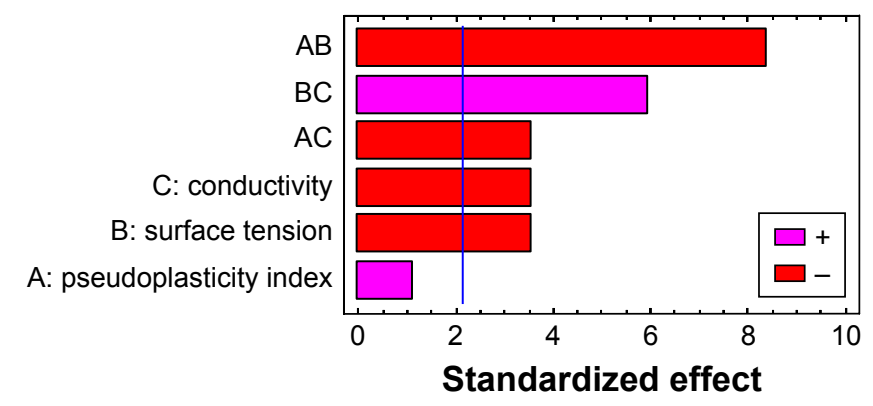

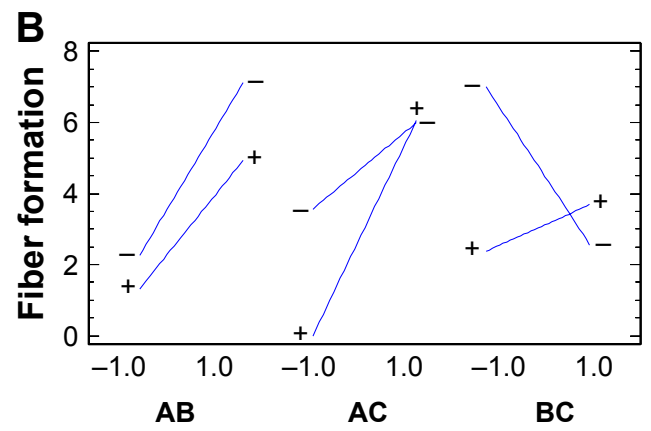

D

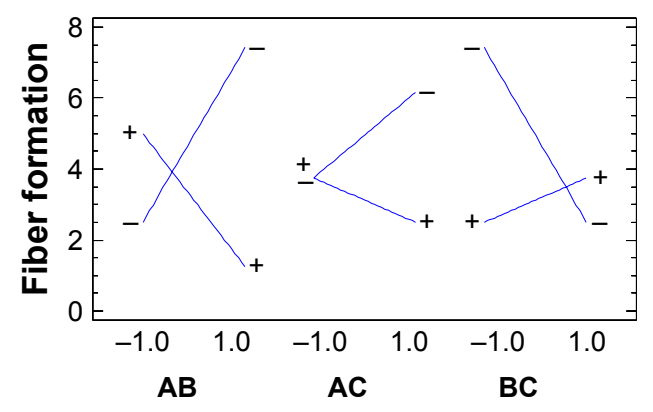

Figure 4 Standardized Pareto chart (A and $\mathbf{C})$ and interaction plot (B and $\mathbf{D})$ of the response variable fiber formation of the full factorial design.

Notes: Three factors, viscosity at high shear rates, surface tension, and conductivity were investigated in $\mathbf{A}$ and $\mathbf{B}$, while pseudoplasticity index, surface tension, and conductivity were investigated in $\mathbf{C}$ and $\mathbf{D}$.

A second full factorial design was performed to investigate the role on fiber morphology of pseudoplasticity index, which is considered in place of viscosity at high shear rates. The interactions of pseudoplasticity index with all the other parameters significantly affect the response variable (Figure 4C). As highlighted by the interaction plot (Figure 4D), the highest response value is obtained when high values of pseudoplasticity index are associated with low values of both surface tension and conductivity.

The DoE approach allows the identification of optimal values for each parameter: high viscosity at $1,000 \mathrm{~s}^{-1}(1.3-1.9$ Pa.s) or a high pseudoplasticity index $\geq 1.7$, associated with a low surface tension $(30-33 \mathrm{mN} / \mathrm{m})$ and a low conductivity $(<1,000 \mu \mathrm{S} / \mathrm{cm})$.

In order to assess the predictive power of the model, two ALG/DEX aqueous mixtures (D9 and D10) having all the parameters in the optimal ranges were prepared and electrospun. Similarly, two ALG/DEX mixtures (D11 and D12) characterized by at least two parameters out of the optimal ranges were considered. In Table 4, the parameters of the above mentioned polymer solutions (D9-D12) are reported. Figure 5 shows the micrographs of the products of the electrospinning process. It can be observed that polymer solutions D9 and D10 determine the formation of homogeneous fibers, whereas fibers with beads are obtained from D11 and D12 polymer solutions.

D9 and D10 fibers with an assigned score of 10 are characterized by diameters of $204 \pm 39$ and $346 \pm 123 \mathrm{~nm}$, respectively (mean values \pm standard error [SE]; $n=30$ ) (Figure 5). Based on these results, D9 fibers are selected for further investigations since they are characterized by the best electrospinning performance (continuous jet formation) and the narrowest size distribution, as evidenced by the lowest value of standard error.

In order to verify whether the guidelines assessed for ALG/DEX mixtures could be suitable also for the production of fibers based on mixtures of ALG with other non-ionic polymers, different from DEX, they were employed for the preparation of ALG/PEO mixtures.

Since DEX shows a rheological behavior, completely different from PEO (Supplementary materials), pseudoplasticity index was considered instead of viscosity as rheological parameter. ALG/PEO mixtures were prepared in order to exhibit parameters in the ranges previously identified as optimal by the DoE approach. Figure 6 reports a SEM micrograph of the optimized ALG/PEO electrospun fibers (P1).

The presence of PEO with high molecular weight $(4,000 \mathrm{kDa})$ in ALG/PEO mixture is responsible for the 
Table 4 Composition (\% w/w) and properties (mean values \pm SD; $n=3$ ) of the polymer solutions D9-DI2 prepared to verify the predictive power of the DoE approach

\begin{tabular}{|c|c|c|c|c|}
\hline & D9 & DIO & DII & DI2 \\
\hline \multicolumn{5}{|l|}{ Composition (\% w/w) } \\
\hline DEX & 30 & 22.5 & 20 & 20 \\
\hline ALG & 0.5 & 1.5 & 1.5 & 1 \\
\hline P407 & 1.5 & 1.5 & 1.5 & 1.5 \\
\hline TX100 & 0.5 & 0.5 & 0.5 & 0.5 \\
\hline \multicolumn{5}{|l|}{ Polymer solution properties } \\
\hline Viscosity (Pa·s) & $1.38 \pm 0.10$ & $1.38 \pm 0.03$ & $0.99 \pm 0.04$ & $0.91 \pm 0.08$ \\
\hline Pseudoplasticity index & $1.791 \pm 0.002$ & $1.923 \pm 0.054$ & $1.790 \pm 0.101$ & $|.589 \pm 0.05|$ \\
\hline Surface tension $(\mathrm{mN} / \mathrm{m})$ & $32.7 \pm 0.8$ & $31.9 \pm 0.6$ & $31.7 \pm 0.4$ & $32.6 \pm 0.5$ \\
\hline Conductivity (mS/cm) & $337 \pm 9$ & $869 \pm 14$ & $\mathrm{I}, 057 \pm \mathrm{I}$ & $836 \pm 3$ \\
\hline
\end{tabular}

Abbreviations: D, ALG/DEX-based solutions; DEX, dextran; ALG, alginate; DoE, Design of Experiments; P407, poloxamer; TXI00, triton.

production of fibers with micro-scale diameter $(13.3 \pm 1.9 \mu \mathrm{m}$, $\mathrm{n}=30$ ).

Mechanical properties of fibers were assessed by means of a tensile test. Maximum force of deformation and forces at different deformation values were measured for the optimized ALG/DEX (D9) and ALG/PEO (P1) fibers. Figure 7 shows an evident break of $\mathrm{D} 9$ fibrous membranes, as indicated by the zero force values for deformations higher than $10 \%$. On the contrary, an elastic deformation characterizes P1 fibers.

D9 and P1 fibers were subjected to ionic cross-linking and/or polymer coating to guarantee their slow biodegradability in biological fluids. In the case of D9, DEX high solubility in both water and organic solvents and high DEX/ $\mathrm{ALG} w / \mathrm{w}$ ratio $(\geq 20)$ do not allow any ionic cross-linking in
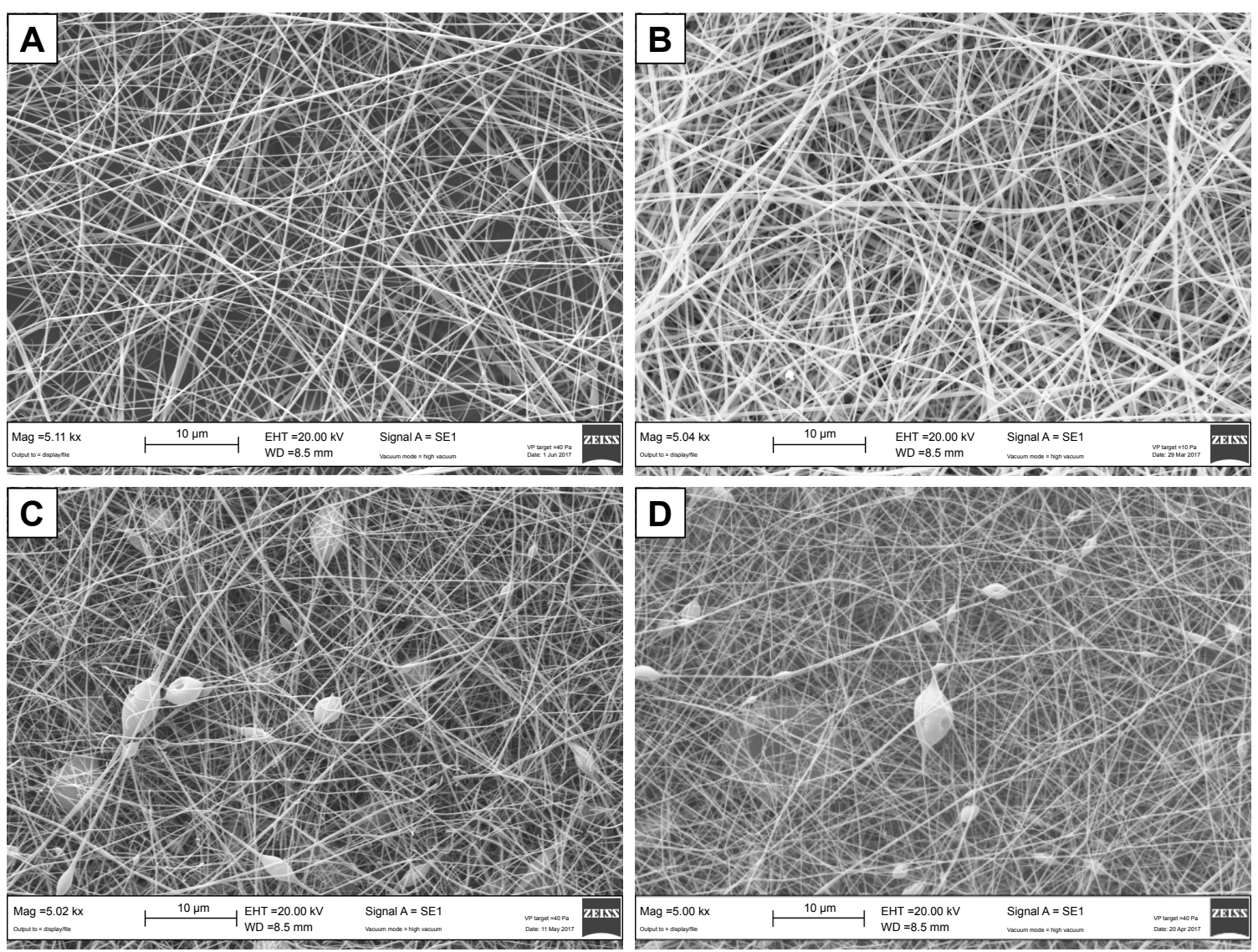

Figure 5 SEM micrographs of (A) D9, (B) DI0, (C) DII, and (D) DI2 products obtained from electrospinning.

Abbreviations: ALG, alginate; D, ALG/DEX-based solutions; DEX, dextran; SEM, scanning electron microscopy. 


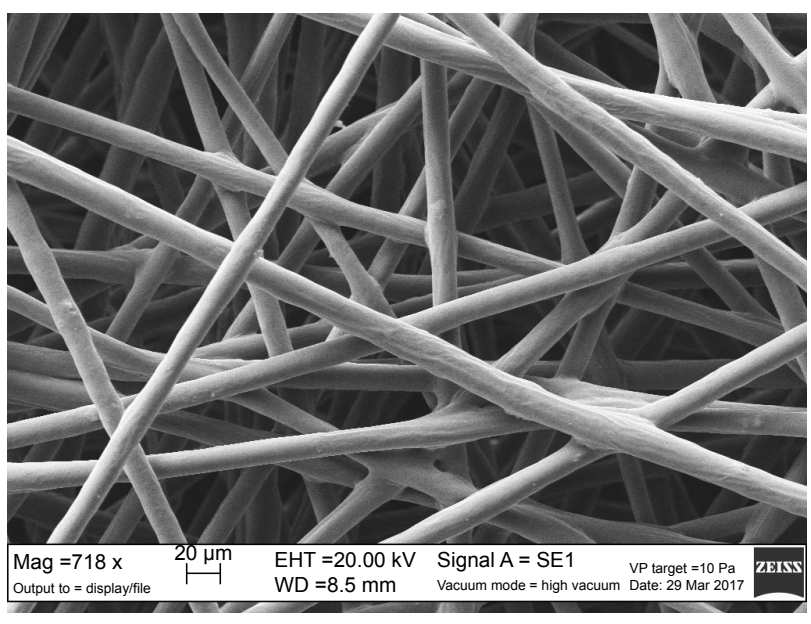

Figure 6 SEM micrograph of the optimized ALG/PEO fibers (PI).

Abbreviations: ALG, alginate; PEO, polyethylene oxide; SEM, scanning electron microscopy.

aqueous/ethanol $\mathrm{CaCl}_{2}$ solutions without the partial loss of membrane integrity. For this reason, D9 fibers were coated by soaking in a PLGA ( $\mathrm{R} 503 \mathrm{H})$ solution in ethyl acetate.

In Figure 8 SEM micrographs, fiber diameters and FTIR spectra of D9 coated with $0.25 \% \mathrm{w} / \mathrm{v}$ and $0.5 \% \mathrm{w} / \mathrm{v} \mathrm{R} 503 \mathrm{H}$ are reported. After coating, a statistically significant increase in fiber diameter is determined, whereas no significant differences in fiber diameter are observed on increasing $\mathrm{R}$ 503H concentration (Figure 8B). FTIR spectra demonstrate the effectiveness of PLGA-based coating on D9 fibers (Figure 8C). FTIR spectrum of uncoated D9 corresponds to DEX one as a consequence of the highest amount of such polysaccharide in comparison with the other components (Table 4). FTIR spectra of coated D9 reveal a peak at $1,756 \mathrm{~cm}^{-1}$ attributable to the presence of PLGA, which, when pure, is characterized by a peak at $1,742 \mathrm{~cm}^{-1}$. The shift observed could be due to a rearrangement of hydrogen bonds after coating. A slight difference between FTIR spectra of D9+ R 503H 0.25\% w/v and D9+ R 503H 0.5\% w/v fibers is attributable to the different PLGA concentrations; in particular, the peak related to $\mathrm{R} 503 \mathrm{H}$ is more evident for D9+ R 503H 0.5\% w/v.

D9 fiber mechanical properties were evaluated before and after coating. In Figure 9, force of deformation, tensile work, and $\% \Delta \mathrm{E}$ of the coated $\mathrm{D} 9$ fibers are compared to those of uncoated ones. PLGA coating produces an increase in fiber mechanical resistance, as evidenced by higher values of both deformation force and tensile work. An increase in PLGA concentration does not produce any change in tensile parameters. The increase in fiber mechanical resistance is accompanied by an increase in fiber elasticity, as indicated by the higher values of $\% \Delta \mathrm{E}$, more evident for the fibers coated with the highest PLGA concentration.

P1 fibers were subjected to a multi-step method that simultaneously provides cross-linking with $\mathrm{Ca}^{2+}$ aqueous/ ethanol solutions and CS-based coating.

Figure 10 reports SEM micrographs, fiber diameters, and FTIR spectra of P1 fibers as such, cross-linked and coated. In the case of coated fibers, a marked surface roughness, functional to cell adhesion, is observed. Figure 10B demonstrates that the cross-linking process produces a statistically significant reduction in ALG/PEO fiber dimension, probably due to the dissolution of PEO and surfactants in water. The FTIR analysis (Figure 10C) confirms the effectiveness of crosslinking and coating procedures by both the disappearance of the peaks related to $\mathrm{PEO}$ and poloxamer and the shift of ALG peak. In particular, FTIR spectra of cross-linked and coated

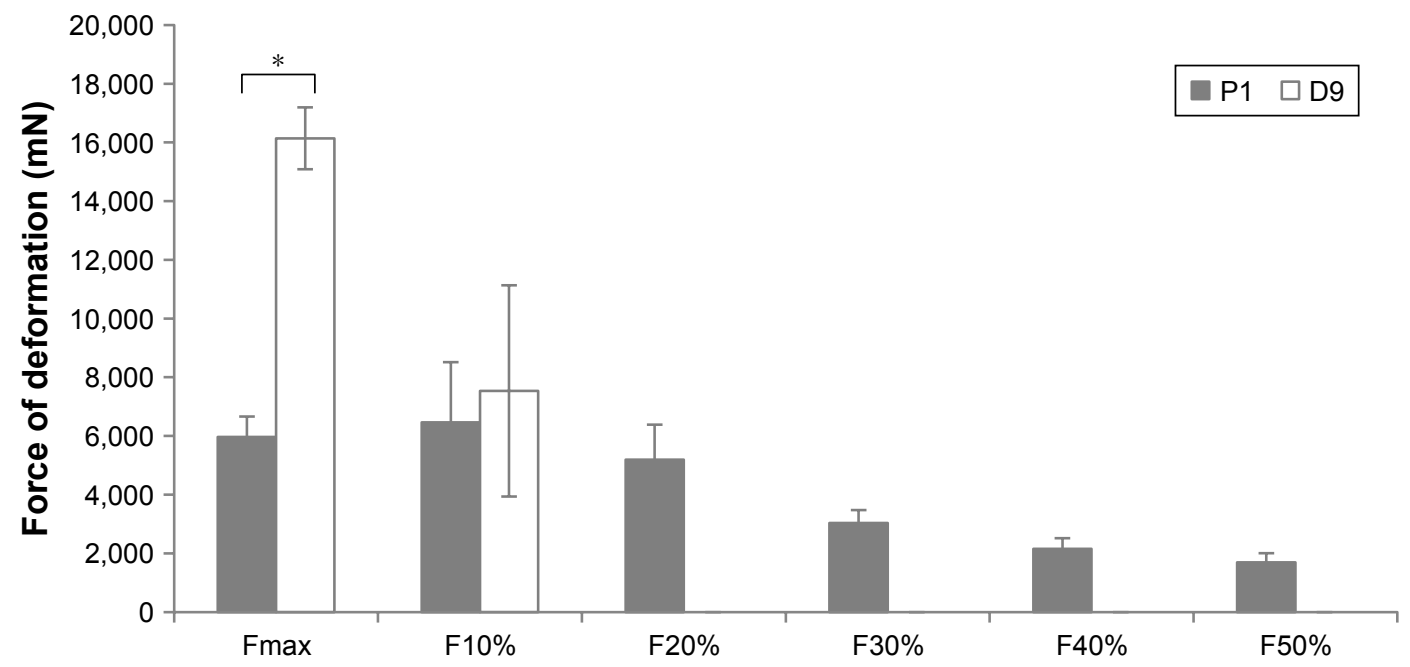

Figure 7 Mechanical properties of both D9 and PI fibers: maximum force of deformation (Fmax) and forces (F) measured at different deformation values are reported (mean values $\pm \mathrm{SE} ; \mathrm{n}=3)$. $* P<0.05$ Student's $t$-test.

Abbreviations: ALG, alginate; D, ALG/DEX-based solutions; P, ALG/PEO fibers; PEO, polyethylene oxide. 
A
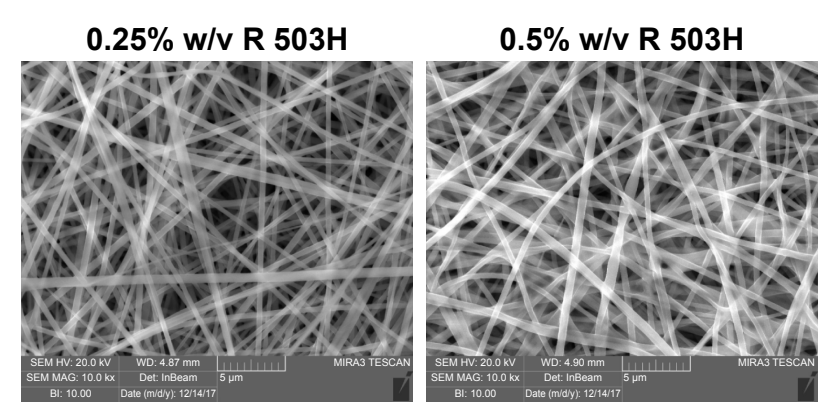

\section{B}

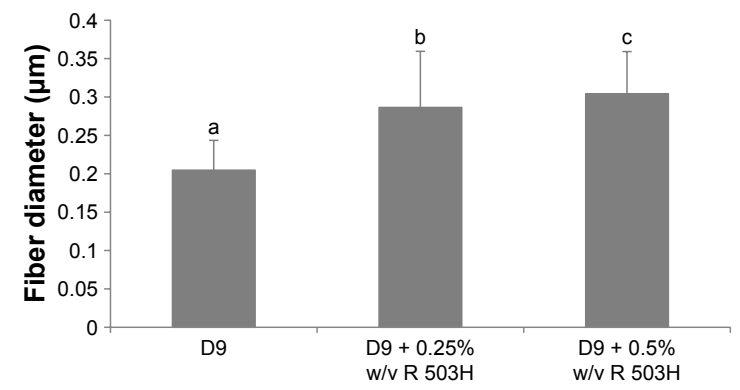

C
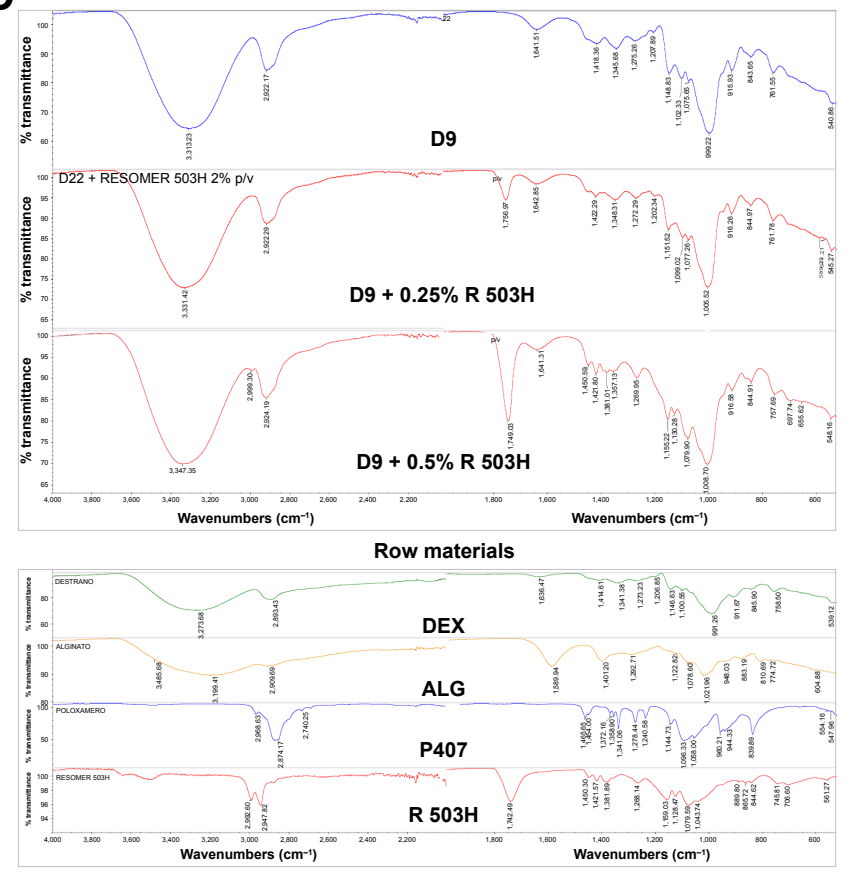

Figure 8 Characterization of D9 fibers after PLGA-based coating: (A) SEM micrographs; (B) fiber dimensions (mean values \pm SD; $n=30$ ), one-way ANOVA, multiple range test $(P<0.05)$ : a vs b and c; $(C)$ FTIR spectrographs.

Abbreviations: ALG, alginate; D, ALG/DEX-based solutions; DEX, dextran; FTIR, Fourier transform infrared; PLGA, polylactide-co-glycolide.

fibers show a shift of peaks corresponding to $-\mathrm{OH}$ bond stretching at 3,246 $\mathrm{cm}^{-1}$ and $\mathrm{C}-\mathrm{O}-\mathrm{C}$ bond stretching at 1,593 and $1,405 \mathrm{~cm}^{-1}$, which demonstrate the occurrence of crosslinking. A slight difference in FTIR spectra of cross-linked and coated fibers could be attributed to the presence of CS on fiber surface, even if at low concentrations. Energy dispersive $\mathrm{X}$-ray spectra of $\mathrm{P} 1$ fibers, cross-linked and coated, before and after hydration in deionized water for 9 hours confirm
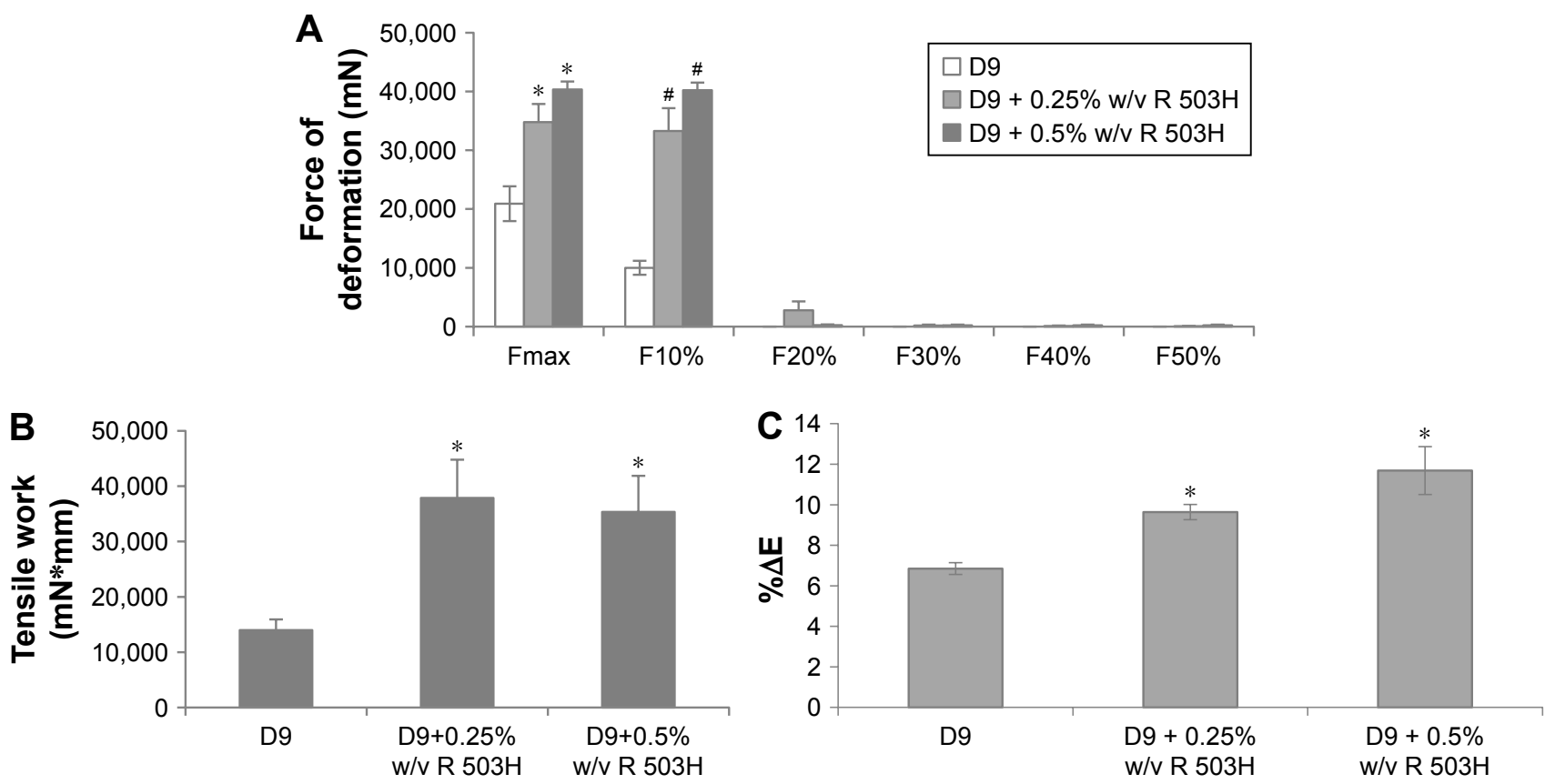

Figure 9 Mechanical properties of D9 as such and after coating with $\mathrm{R} 503 \mathrm{H}$ at $0.25 \%$ and $0.5 \% \mathrm{w} / \mathrm{v}$ : (A) maximum force of deformation (Fmax) and forces (F) measured at different deformation values ( $* P<0.05$ compared to D9 Fmax and ${ }^{*} P<0.05$ compared to D9 FI0\%, Student's t-test), (B) tensile work ( $* P<0.05$ compared to D9, Student's $t$-test), and $(\mathbf{C})$ percentage of fiber elongation ( $* P<0.05$ compared to $D 9)$ are reported (mean values $\pm S E ; n=3$ ).

Abbreviations: ALG, alginate; D, ALG/DEX-based solutions; DEX, dextran. 
A

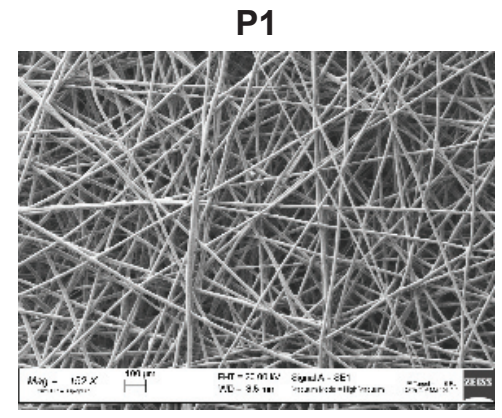

P1 cross-linked

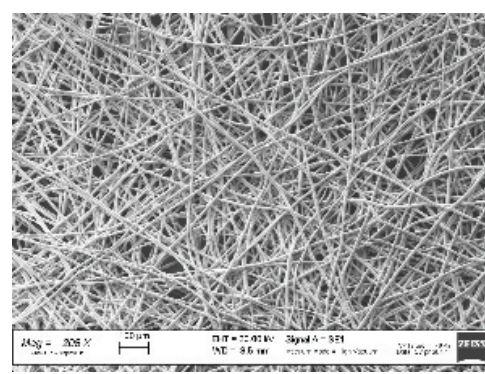

\section{P1 coated}

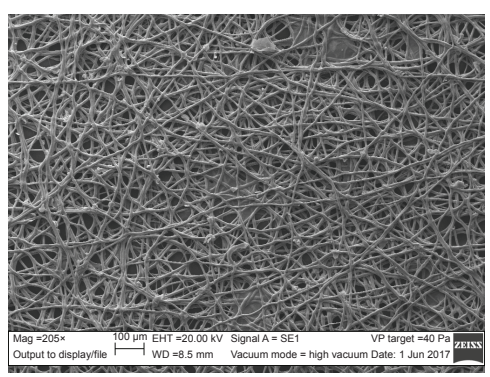

\section{B}

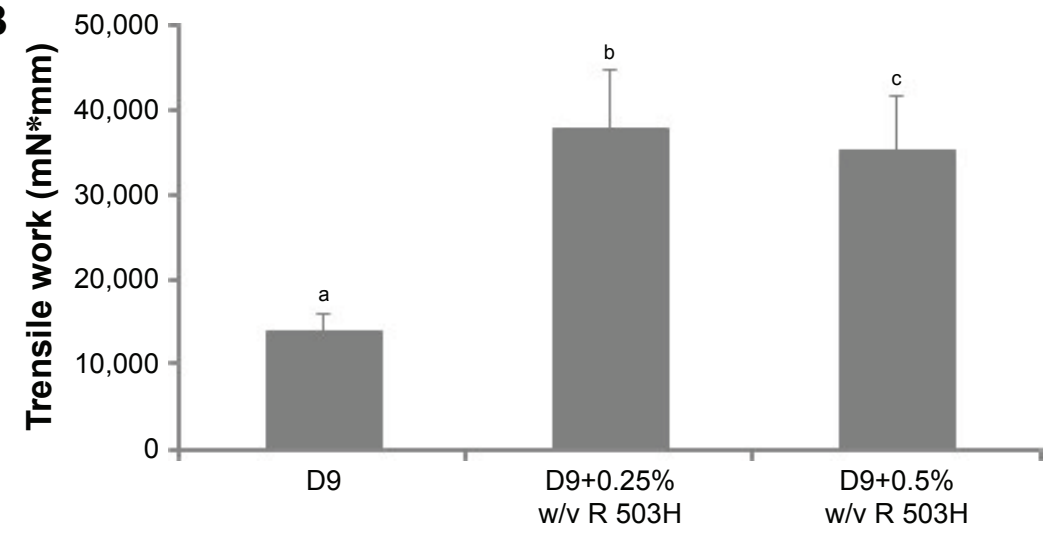

C
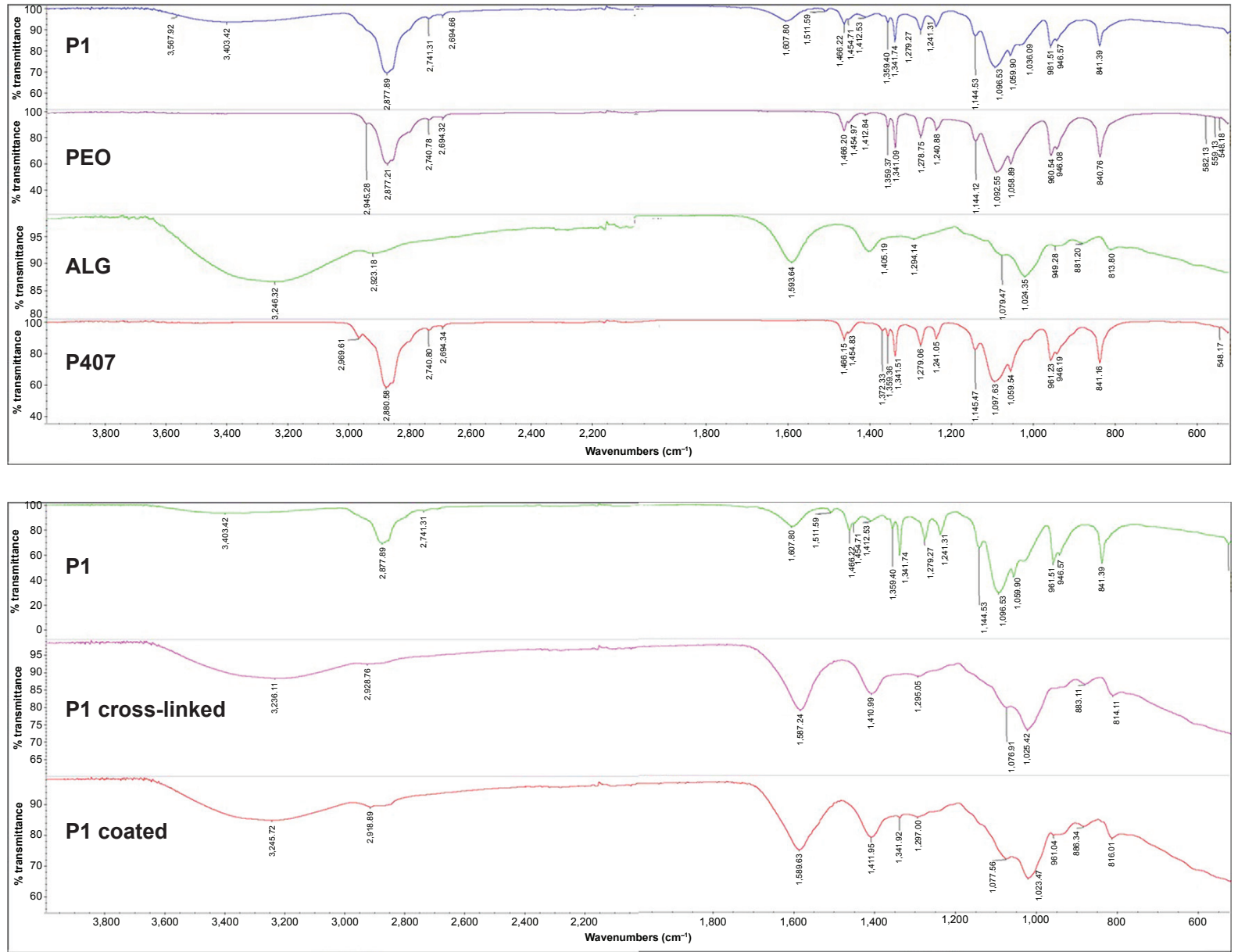

Figure 10 Characterization of $\mathrm{PI}$ fibers after $\mathrm{Ca}^{2+}$ cross-linking and chitosan-based coating: (A) SEM micrographs; (B) fiber dimensions (mean \pm SD; $\mathrm{n}=30$ ), one-way ANOVA, multiple range test $(P<0.05)$ : a vs b and c; (C) FTIR spectrographs.

Abbreviations: ALG, alginate; D, ALG/DEX-based solutions; P, ALG/PEO fibers; PEO, polyethylene oxide; SEM, scanning electron microscopy; FTIR, Fourier transform infrared. 
cross-linking of fibers (Table 5). The increase of $\mathrm{O} \%$ is to be attributed to fiber hydration and swelling.

In Figure 11, force of deformation, tensile work, and $\% \Delta \mathrm{E}$ of cross-linked and coated $\mathrm{P} 1$ fibers are compared with those of untreated $\mathrm{P} 1$ fibers. Both cross-linking and coating processes are responsible for an increase in fiber mechanical resistance, as indicated by higher values of maximum force of deformation (Figure 11A). Cross-linked fibers show a less elastic structure (evidenced by a lower value of tensile work, Figure 11B), due to PEO elimination during cross-linking. Coating with CS improves fiber mechanical properties in terms of resistance and elasticity, as indicated by the increase of all the tensile parameters.

The biocompatibility and the capability to support cell adhesion and proliferation were evaluated for both PLGA-coated D9 and CS-coated P1 fibers on human dermal fibroblasts.

In Figure 12, \% cell viability values observed for coated D9 and P1 fibers are reported. Both fibrous platforms showed $\%$ cell viability values comparable to those obtained for CM, used as reference. Such results indicate the biocompatibility of both the formulations.

Figure 13 shows SEM micrographs of coated D9 and P1 fibers after immersion in CM for 7 days in the absence and in the presence of fibroblasts. It can be observed that, after hydration in CM, coated D9 fibers appear as a micro-porous matrix (Figure 13A); the comparison between Figure A and B evidence the presence of fibroblasts on the fibrous membrane.

After 7 days, cells appear well extended within the structure of P1 coated fibers, showing their typical elongated shape (C-E). This result could be related to the surface properties of CS-coated P1 fibers, which appear suitable to promote cell adhesion and growth. At the end of the experiment, a great amount of cells are observed at the well bottom. This is probably due to the high porosity (pore diameter $=43 \pm 3.2$ $\mu \mathrm{m}$ [mean value $\pm \mathrm{SE} ; \mathrm{n}=30]$ ) of the fibrous membrane, which enables cell filtration and growth at the well bottom.

\section{Discussion}

In the present work, DEX and PEO were used to enhance ALG electrospinnability. In the past, Nie et al proved that a little amount of PEO with $\mathrm{MW}=1,000 \mathrm{kDa}$ improved the electrospinnability of ALG aqueous solutions. ${ }^{18}$ Such a behavior was determined by the fact that PEO offers entanglement sites and, thereby, is capable of enhancing the entanglement degree of the mixture. Caykara et al effected FTIR studies on ALG/PEO blend-based films prepared by casting. ${ }^{30}$ They observed the formation of hydrogen bonds between PEO ether oxygen and ALG hydroxyl groups. ALG/PEO blend-based films were characterized by improved mechanical properties when compared with those of the two homopolymers. These results were attributed to intermolecular hydrogen bonding between ALG and PEO.

According to Nie et al, the rigidity of ALG chains is mainly caused by $\mathrm{G}$ units, which are linked by diaxial linkages and stabilized by hydrogen bonding. ${ }^{17}$ The rigidity, together with the extended worm-like molecular structure, is responsible for the lack of effective chain entanglements in aqueous solution. The authors proved with viscoelastic measurements that the introduction of glycerol into ALG aqueous solution changes ALG chain conformation, which results in improved chain flexibility and entanglement. Glycerol, possessing three hydroxyl groups per molecule, can interact with ALG by disrupting a large number of inter- and intra-molecular hydrogen bonds of ALG chains and by forming ALG-glycerol hydrogen bonds. The same mechanism can be assumed for ALG/DEX mixtures.

To verify such hypothesis, mixtures of DEX 30\% w/w with increasing ALG concentrations $(0.5 \%$ w/w ALG and $30 \% \mathrm{w} / \mathrm{w} \mathrm{DEX}$ ) were subjected to viscoelastic measurements and the differential parameter $\Delta \mathrm{G}^{\prime}$ was calculated for each mixture. According to de Souza Ferreira et al, such parameter has to be considered as index of the extent of the polymer interaction. ${ }^{27}$ The positive values of $\Delta G^{\prime}$ observed for DEX/ ALG mixtures indicate the occurrence of an interaction between the two polymers, presumably due to the formation of hydrogen bonds. Such interaction is responsible for an increase in polymer network elasticity. The increase in ALG concentration produces the formation of a higher number of bonds, resulting in a strengthening of the polymeric network that, consequently, is characterized by a higher elasticity (Figure 2). The resulting increase in chain entanglement is functional to the formation of fibers by electrospinning and explains the role of DEX as ALG adjuvant.

Polymer solution properties (such as viscosity, surface tension, and conductivity) as well as process (applied voltage,

Table 5 Element content (mol \%) of coated PI fibers before and after hydration in deionized water

\begin{tabular}{|c|c|c|c|c|c|}
\hline Sample & C & $\mathbf{0}$ & $\mathbf{C a}$ & $\mathbf{C l}$ & Other elements \\
\hline $\mathrm{PI}$ & 51.26 & 21.82 & 0 & 0 & 26.92 \\
\hline PI coated & 30.91 & 32 & 25.65 & 0.23 & 5.21 \\
\hline $\mathrm{PI}$ coated after hydration in deionized $\mathrm{H}_{2} \mathrm{O}$ & 35.02 & $4 I .4 I$ & 17.88 & 0 & 5.69 \\
\hline
\end{tabular}

Note: $\mathrm{PI}$ fibers as such was reported as reference.

Abbreviations: ALG, alginate; P, ALG/PEO fibers; PEO, polyethylene oxide. 
A

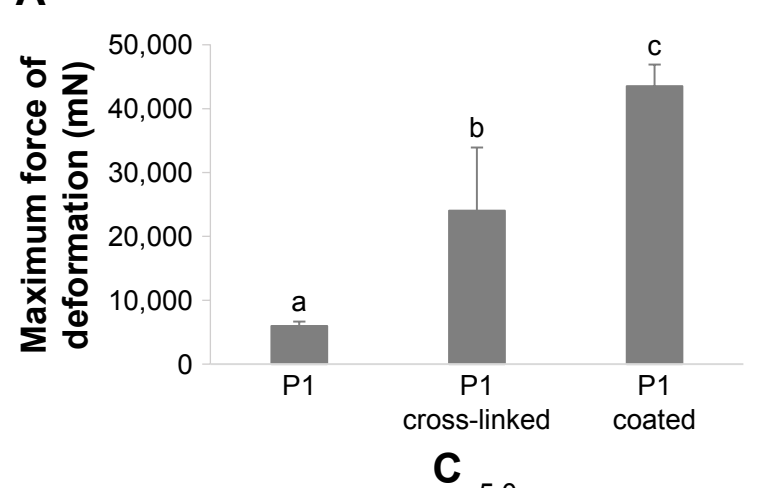

B

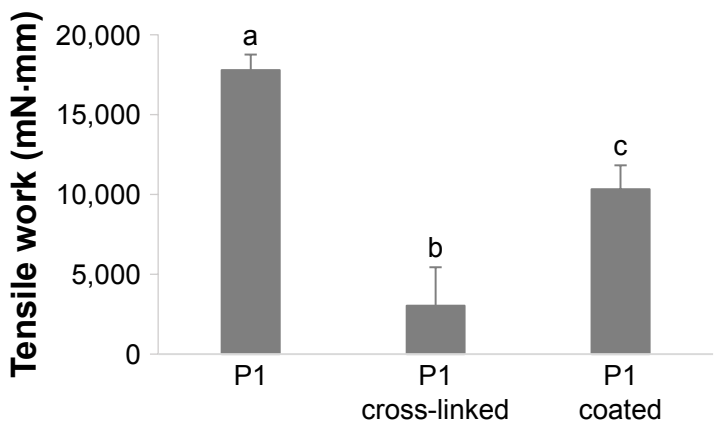

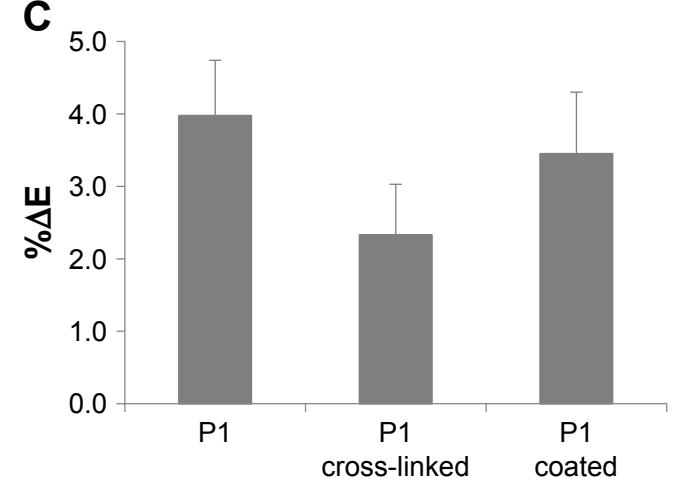

Figure I I Mechanical properties of PI fibers as such and after $\mathrm{Ca}^{2+}$ cross-linking and chitosan-based coating: (A) maximum force of deformation (one-way ANOVA, multiple range test $[P<0.05]$ : a vs b and c; b vs $c$ ), (B) tensile work (one-way ANOVA, multiple range test $[P<0.05]$ : $a$ vs b and c; b vs $c$ ), and $(\mathbf{C})$ percentage of fiber elongation are reported (mean values $\pm S E ; n=3$ ).

Abbreviations: ALG, alginate; P, ALG/PEO fibers; PEO, polyethylene oxide.

spinneret-collector distance, and flow rate) and environmental (temperature and relative humidity) parameters strongly affect jet formation and can be opportunely modulated in order to obtain fibers of desired morphology and diameter. ${ }^{10,11,25}$

The identification of the quantitative composition of the polymer solution to be electrospun is generally time consuming. A trial and error approach is usually employed, and several attempts are required to produce homogeneous fibers. The DoE approach proposed in this study allows to draw

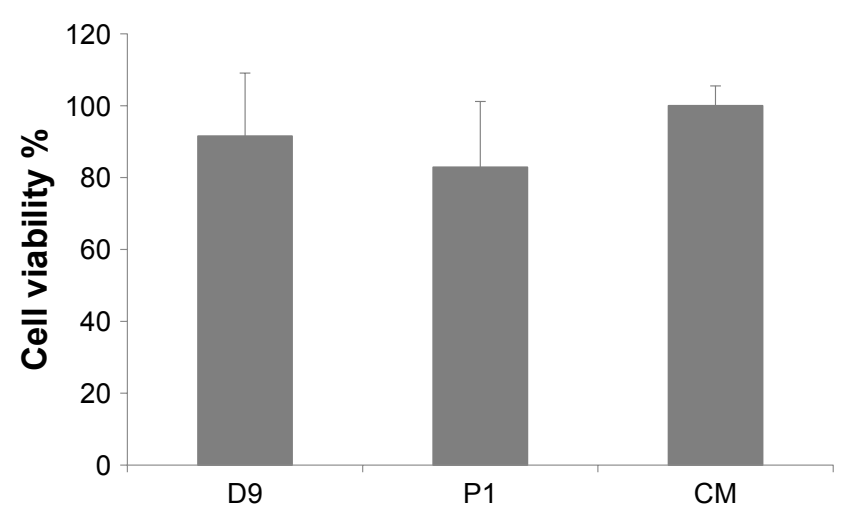

Figure $12 \%$ Cell viability values observed for coated D9 and PI fibers (mean values $\pm S D ; n=6$ ).

Abbreviations: ALG, alginate; CM, complete medium; D, ALG/DEX-based solutions; DEX, dextran; P, ALG/PEO fibers; PEO, polyethylene oxide. up guidelines to be used for the preparation of mixtures of ALG with non-ionic polymers able to produce homogeneous nanofibers by electrospinning.

The first full factorial design employed proves that the solution (D5), where DEX is present in a concentration lower than CEC, does not produce fibers. This result confirms the findings of McKee et al, who demonstrated that any polymer solutions could produce electrospun homogeneous fibers when prepared at polymer concentrations $2-8$ times above the CEC, thus ensuring a sufficient chain overlap. ${ }^{31}$

The results reported in the Pareto charts evidence the negative effects of both conductivity and surface tension. Conductivity is related to the number of electric charges present on the surface of the polymer solution to be electrospun and affects its capability to form nanofibers. The electrospinning process needs a minimum electrical conductivity of the polymer solution: a polymer solution lacking in charges cannot be electrospun. ${ }^{32}$ In fact, an electrical charge transfer from the electrode to the polymer droplet located at the ending point of the injection needle is required. Generally, the increase in electrical charge determines an increase in the elongation capacity of the solution, favoring the formation of smooth fibers, with smaller diameter. ${ }^{32}$ Other studies suggest that an increase in solution conductivity leads to the production of homogenous nanofibers. ${ }^{10}$ 


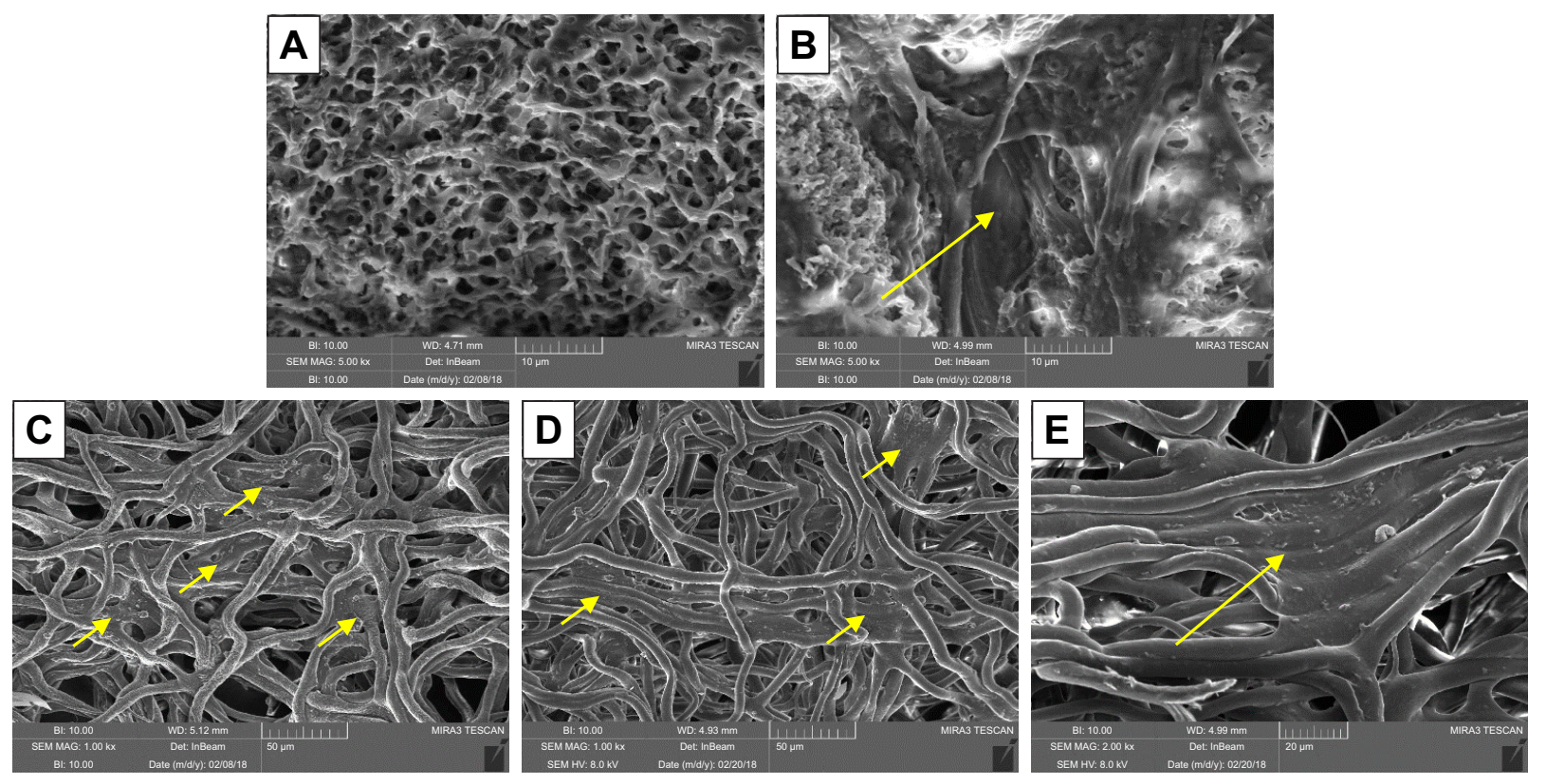

Figure 13 SEM micrographs of the coated fibers: (A, B) coated D9 fibers after immersion for 7 days in complete medium in the absence (A) and in the presence (B) of fibroblasts; (C-E) coated PI fibers after immersion for 7 days in complete medium in the presence of fibroblasts. The yellow arrows indicate cells. Abbreviations: ALG, alginate; D, ALG/DEX-based solutions; P, ALG/PEO fibers; PEO, polyethylene oxide; SEM, scanning electron microscopy.

DoE results obtained in the present work seem in disagreement with that reported in literature. ${ }^{10,32}$ It must be underlined that the conductivity of a polymer solution depends not only on the solvent used but also on the amount and distribution of polymer surface charges. Therefore, the influence of conductivity has to be considered case by case. In the present paper, the optimal range of conductivity associated with the formation of homogeneous fibers is found when ALG is mixed with a non-ionic polymer.

Surface tension is another important parameter, since it strictly affects the formation of Taylor's cone during the electrospinning process. ${ }^{10}$ Surface tension can be easily changed by varying the composition of the solvent. It is generally recognized that a decrease of surface tension enables fiber formation at lower electric fields. In the present work, the surface tension of the various polymer solutions has been modulated by adding different types and concentrations of surfactants such as Poloxamer and Triton.

A high viscosity at high shear rates promotes the formation of bead-free fibers, as confirmed by other works, reported in literature, which demonstrated that low viscosity of polymer solution could determine the disruption of the polymeric jet, which, in turn, causes bead formation within the fibers. ${ }^{33,34}$

In the second full factorial design, viscosity at high shear rates was substituted by the pseudoplasticity index. To the best of our knowledge, the influence of such a factor on the production of homogeneous fibers has not yet been thoroughly investigated. Pseudoplasticity index takes into account the whole viscosity profile: high values of pseudoplasticity index indicate a marked difference between viscosity at rest $\left(\eta_{0}\right)$ and at high shear $\left(\eta_{\infty}\right)$. The results indicate that high pseudoplasticity index values, when associated with low surface tension and conductivity values, lead to homogeneous fiber formation.

It must be underlined that, for all the polymer solutions that failed in the production of fiber, such event was independent of the electrospinning parameters and, in the case of polymer solution having optimized properties, the modulation of process parameters was adopted to obtain an improved fiber yield.

The aim of this study was to set guidelines that could be exploited for the production of fibers containing ALG mixed with non-ionic polymers. Therefore, optimal ranges found for the production of ALG/DEX fibers were employed for the preparation of ALG/PEO ones. Since DEX and PEO showed different viscosity behavior (Supplementary materials), pseudoplasticity index was retained as rheological parameter.

An ALG/PEO mixture (P1) having pseudoplasticity index, conductivity, and surface tensions within the ranges identified by the DoE approach was prepared and electrospun. It produced homogeneous fibers, proving the goodness of the guidelines.

D9 and P1 fibers were compared for size and mechanical resistance. D9 fibers showed nanoscale dimensions and resulted brittle, while P1 fibers were characterized by microsize and elastic deformation. 
Both the fibrous platforms were subsequently crosslinked and/or coated to guarantee their slow biodegradability in biological fluids. Two different methods were used for D9 and P1 fibers, taking into account the solubility of each adjuvant polymer, DEX and PEO.

The few papers, in literature, dealing with the use of DEX for the production of electrospun fibers, combined this polysaccharide with other synthetic polymer, such as polyurethane ${ }^{4}$ or subjected DEX to modifications by substitution of methacrylate groups at the hydroxyl sites, followed by photo-cross-linking. ${ }^{21}$ In the present work, D9 fibers were coated with PLGA, which produces an increase in fiber dimensions and mechanical resistance. In particular, an increase in fiber elasticity is observed; it depends on PLGA concentration.

It is well-known that ALG undergoes cross-linking in the presence of divalent cations, such as $\mathrm{Ca}^{2+}$, which determines hydrogel structures with a controlled degradation rate. In particular, ALG carboxylic groups interact with cations causing bridge formation between polymer chains. ${ }^{20}$ ALG is also able to interact with the cationic CS. The partial protonated amine groups of CS react with the carboxylate groups of ALG and thus strong polyelectrolyte complexes are formed. ${ }^{35,36} \mathrm{P} 1$ fibers were simultaneously cross-linked/ coated with calcium ions and CS. During cross-linking, fiber soaking into aqueous solutions causes the removal of PEO and surfactant. The presence of CS determines an increase in fiber elastic properties, which are partly lost due to the disappearance of PEO.

Both coated D9 and P1 fibers result to be biocompatible and able to support cell adhesion and proliferation.

SEM analysis show that, after soaking in $\mathrm{CM}$ for 7 days, PLGA-coated D9 fibers appear as a micro-porous matrix without any sign of degradation. Such a result is in agreement with literature findings. It is well known that the hydrophobicity and then the degradation time of PLGA could be modulated by varying the lactide (L)-to-glycolide (G) ratio. ${ }^{37,38}$

As for coated P1 fibers, several papers reported in literature confirm that ALG-based scaffolds, after cross-linking with bivalent ions, for example calcium ones, maintain their integrity, both in vitro and in vivo, up to 30 days. In particular, both $\mathrm{ALG}$ and $\mathrm{Ca}^{2+}$ concentrations seem to play a pivotal role in determining scaffold degradation rate. ${ }^{39}$ These results were confirmed by Vigani et al $;{ }^{40}$ they observed that ALG-calcium beads, when implanted in the subcutaneous tissue of a murine model, preserved their structure for 30 days. ALG-calcium beads were no longer found after 60 days: the authors supposed that such beads dissolve due to an exchange reaction between calcium and sodium ions at the implantation site. ${ }^{40}$

Rong et al investigated in vivo degradation behavior of thrombin-loaded ALG-calcium microspheres after subcutaneous implantation in rats, revealing, through histological analyses, that only at 10 weeks it was not possible to identify microspheres anymore. ${ }^{41}$

The biodegradation rate of coated P1 fibers depends not only on calcium-cross-linking of ALG but also on the ionic interaction between the carboxylate moieties of ALG and the protonated amines on CS. The soaking of P1 fibers in a $\mathrm{CaCl}_{2} / \mathrm{CS}$ solution promotes the formation of $\mathrm{ALG}-\mathrm{CS}$ interactions, which have been reported to slow down polymer matrix degradation. ${ }^{36}$

Since fiber biodegradation depends not only on the intrinsic properties of the coating polymers but also on fiber morphology and inner composition, studies are in progress to assess coated D9 and P1 degradation in physiological media.

\section{Conclusion}

The DoE approach proposed in this study allows to draw up guidelines, which are useful for the preparation of mixtures of ALG with non-ionic polymers that are able to produce homogeneous fibers by electrospinning. In particular, optimal values for each property are identified.

Both ALG/PEO and ALG/DEX fibers are successfully coated with CS and PLGA, respectively. Coating process improves fiber mechanical properties and slows down their biodegradation in physiological medium. The developed fibrous platforms are biocompatible and able to support cell growth.

\section{Acknowledgment}

The authors would like to thank Dr Ilenia Tredici, CISRiC Arvedi, Pavia University, for her assistance in EDS and SEM analyses.

\section{Author contributions}

All authors contributed toward data analysis, drafting and critically revising the paper, gave final approval of the version to be published, and agree to be accountable for all aspects of the work. SR and BV conceived and designed the experiments and wrote the paper. GM performed the experiments. GB performed SEM and FTIR analyses. GS, MCB, and FF reviewed the manuscript.

\section{Disclosure}

The authors report no conflicts of interest in this work. 


\section{References}

1. Cao H, Liu T, Chew SY. The application of nanofibrous scaffolds in neural tissue engineering. Adv Drug Deliv Rev. 2009;61(12):1055-1064.

2. Ma B, Xie J, Jiang J, Shuler FD, Bartlett DE. Rational design of nanofiber scaffolds for orthopedic tissue repair and regeneration. Nanomedicine. 2013;8(9):1459-1481.

3. Chou SF, Carson D, Woodrow KA. Current strategies for sustaining drug release from electrospun nanofibers. J Control Release. 2015; 220(Pt B):584-591.

4. Unnithan AR, Barakat NA, Pichiah PB, et al. Wound-dressing materials with antibacterial activity from electrospun polyurethane-dextran nanofiber mats containing ciprofloxacin HCl. Carbohydr Polym. 2012;90(4):1786-1793.

5. Kamble P, Sadarani B, Majumdar A, Bhullar S. Nanofiber based drug delivery systems for skin: A promising therapeutic approach. J Drug Deliv Sci Technol. 2017;41:124-133.

6. Janković B, Pelipenko J, Škarabot M, Muševič I, Kristl J. The design trend in tissue-engineering scaffolds based on nanomechanical properties of individual electrospun nanofibers. Int J Pharm. 2013; 455(1-2):338-347.

7. Raspa A, Marchini A, Pugliese R, et al. A biocompatibility study of new nanofibrous scaffolds for nervous system regeneration. Nanoscale. 2016;8(1):253-265.

8. Hu X, Liu S, Zhou G, Huang Y, Xie Z, Jing X. Electrospinning of polymeric nanofibers for drug delivery applications. J Control Release. 2014;185:12-21.

9. Abrigo M, Mcarthur SL, Kingshott P. Electrospun nanofibers as dressings for chronic wound care: advances, challenges, and future prospects. Macromol Biosci. 2014;14(6):772-792.

10. Rogina A. Electrospinning process: Versatile preparation method for biodegradable and natural polymers and biocomposite systems applied in tissue engineering and drug delivery. Appl Surf Sci. 2014;296:221-230.

11. Bhardwaj N, Kundu SC. Electrospinning: a fascinating fiber fabrication technique. Biotechnol Adv. 2010;28(3):325-347.

12. Vigani B, Rossi S, Sandri G, Bonferoni MC, Ferrari F. Design and criteria of electrospun fibrous scaffolds for the treatment of spinal cord injury. Neural Regen Res. 2017;12(11):1786-1790.

13. Lee KY, Mooney DJ. Alginate: properties and biomedical applications. Prog Polym Sci. 2012;37(1):106-126.

14. Bhattarai N, Li Z, Edmondson D, Zhang M. Alginate-based nanofibrous scaffolds: structural, mechanical, and biological properties. Adv Mater. 2006;18(11):1463-1467.

15. Jw L, Zhu YL, Guo ZX, Hu P, Yu J. Electrospinning of sodium alginate with poly(ethylene oxide). Polymer. 2006;47:8026-8031.

16. Safi S, Morshed M, Hosseini Ravandi SA, Ghiaci M. Study of electrospinning of sodium alginate, blended solutions of sodium alginate/ poly(vinyl alcohol) and sodium alginate/poly(ethylene oxide). J Appl Polym Sci. 2007;104(5):3245-3255.

17. Nie H, He A, Zheng J, Xu S, Li J, Han CC. Effects of chain conformation and entanglement on the electrospinning of pure alginate. Biomacromolecules. 2008;9(5):1362-1365.

18. Nie H, He A, Wu W, et al. Effect of poly(ethylene oxide) with different molecular weights on the electrospinnability of sodium alginate. Polymer. 2009;50(20):4926-4934.

19. Bonino CA, Krebs MD, Saquing CD, et al. Electrospinning alginatebased nanofibers: From blends to crosslinked low molecular weight alginate-only systems. Carbohydr Polym. 2011;85(1):111-119.

20. Lee KY, Jeong L, Kang YO, Lee SJ, Park WH. Electrospinning of polysaccharides for regenerative medicine. Adv Drug Deliv Rev. 2009; 61(12):1020-1032.

21. Jiang H, Fang D, Hsiao BS, Chu B, Chen W. Optimization and characterization of dextran membranes prepared by electrospinning. Biomacromolecules. 2004;5(2):326-333.
22. Unnithan AR, Sasikala AR, Murugesan P, et al. Electrospun polyurethane-dextran nanofiber mats loaded with Estradiol for post-menopausal wound dressing. Int J Biol Macromol. 2015;77:1-8.

23. Deitzel J, Kleinmeyer J, Hirvonen JK, Kang MG. Controlled deposition of electrospun poly(ethylene oxide) fibers. Polymer. 2001;42(19): 8163-8170.

24. Francis R, Kumar DS, editors. Biomedical Applications of Polymeric Materials and Composites. Weinheim: John Wiley \& Sons; 2016.

25. Haider A, Haider S, Kang I-K. A comprehensive review summarizing the effect of electrospinning parameters and potential applications of nanofibers in biomedical and biotechnology. Arab J Chem. Epub 12 Dec 2015.

26. Hashemi Gahruie H, Niakousari M, Antioxidant NM. Antioxidant, antimicrobial, cell viability and enzymatic inhibitory of antioxidant polymers as biological macromolecules. Int J Biol Macromol. 2017; 104(Pt A):606-617.

27. de Souza Ferreira SB, da Silva JB, Borghi-Pangoni FB, Junqueira MV, Bruschi ML. Linear correlation between rheological, mechanical and mucoadhesive properties of polycarbophil polymer blends for biomedical applications. J Mech Behav Biomed Mater. 2017;68:265-275.

28. Shao P, Qin M, Han L, Sun P. Rheology and characteristics of sulfated polysaccharides from chlorophytan seaweeds Ulva fasciata. Carbohydr Polym. 2014;113:365-372.

29. Rezvanian M, Ahmad N, Mohd Amin MC, Ng SF. Optimization, characterization, and in vitro assessment of alginate-pectin ionic cross-linked hydrogel film for wound dressing applications. Int J Biol Macromol. 2017;97:131-140.

30. Çaykara T, Demirci S, Eroğlu MS, Güven O. Poly(ethylene oxide) and its blends with sodium alginate. Polymer. 2005;46(24):10750-10757.

31. Mckee MG, Hunley MT, Layman JM, Long TE. Solution Rheological Behavior and Electrospinning of Cationic Polyelectrolytes. Macromolecules. 2006;39(2):575-583.

32. Huang Z-M, Zhang Y-Z, Kotaki M, Ramakrishna S. A review on polymer nanofibers by electrospinning and their applications in nanocomposites. Compos Sci Technol. 2003;63(15):2223-2253.

33. Zhu J, Shao H, Hu X. Morphology and structure of electrospun mats from regenerated silk fibroin aqueous solutions with adjusting $\mathrm{pH}$. Int J Biol Macromol. 2007;41(4):469-474.

34. Huang Z-M, Zhang YZ, Ramakrishna S, Lim CT. Electrospinning and mechanical characterization of gelatin nanofibers. Polymer. 2004;45(15):5361-5368.

35. Conzatti G, Faucon D, Castel M, Ayadi F, Cavalie S, Tourrette A. Alginate/chitosan polyelectrolyte complexes: A comparative study of the influence of the drying step on physicochemical properties. Carbohydr Polym. 2017;172:142-151.

36. Li X, Xie H, Lin J, Xie W, Ma X. Characterization and biodegradation of chitosan-alginate polyelectrolyte complexes Poly Degrad Stab. 2009;94(1):1-6.

37. Liu C, Wang C, Zhao Q, et al. Incorporation and release of dual growth factors for nerve tissue engineering using nanofibrous bicomponent scaffolds. Biomed Mater. 2018;13(4):044107.

38. Rao J, Cheng Y, Liu Y, et al. A multi-walled silk fibroin/silk sericin nerve conduit coated with poly(lactic-co-glycolic acid) sheath for peripheral nerve regeneration. Mater Sci Eng C Mater Biol Appl. 2017; 73:319-332.

39. Lee KY, Mooney DJ. Alginate: properties and biomedical applications. Prog Polym Sci. 2012;37(1):106-126.

40. Vigani B, Mastracci L, Grillo F, et al. Local biological effects of adipose stromal vascular fraction delivery systems after subcutaneous implantation in a murine model. J Bioact Compat Polym. 2016; 31(6):600-612.

41. Rong JJ, Liang M, Xuan FQ, et al. Alginate-calcium microsphere loaded with thrombin: a new composite biomaterial for hemostatic embolization. Int J Biol Macromol. 2015;75:479-488. 


\section{Supplementary materials}

Assessment of viscoelastic properties of alginate/dextran (ALG/DEX) mixture

In Figure $S 1$, storage elastic modulus $\left(\mathrm{G}^{\prime}\right)$ vs frequency profiles of ALG-DEX mixtures in comparison with those obtained for DEX and ALG solutions are reported. It can be observed that the mixtures were characterized by $\mathrm{G}^{\prime}$ values markedly higher than those of the individual polymer solutions.

\section{Assessment of critical entanglement concentration (CEC) of DEX}

In the present study, DEX CEC was evaluated. Briefly, different DEX solutions with increasing polymer concentrations, ranging from $0.5 \%$ to $40 \% \mathrm{w} / \mathrm{w}$, were prepared in deionized water and in $\mathrm{pH} 5.5$ phosphate buffer. Flow curves, reporting shear stress $(\tau)$ versus shear rate, were obtained for each DEX solution by means of a rotational rheometer (MCR $102)$, using a $C 50-1$ cone $\left(\varnothing=50 \mathrm{~mm}\right.$ and $\left.\vartheta=1^{\circ}\right)$ as measuring system. Measurements were performed in a shear rate ranging from 10 to $1,000 \mathrm{~s}^{-1}$ at $33^{\circ} \mathrm{C}$ (temperature to which the polymer solutions were subjected during the electrospinning process). Viscosity values at rest $\left(\eta_{0}\right)$ and at high shear $\left(\eta_{\infty}\right)$ were calculated from the slopes of the straight lines that fitted the experimental data of the first and the second Newtonian zones of the flow curve. $\eta_{0}$ and $\eta_{\infty}$ were plotted versus the corresponding polymer concentration $(\% \mathrm{w} / \mathrm{w})$ on bi-log scale. The intersection point of the two straight lines corresponded to $\mathrm{CEC}$. $^{1}$

The CEC represents the lowest concentration at which the entanglement of the polymeric chains occurs; it depends

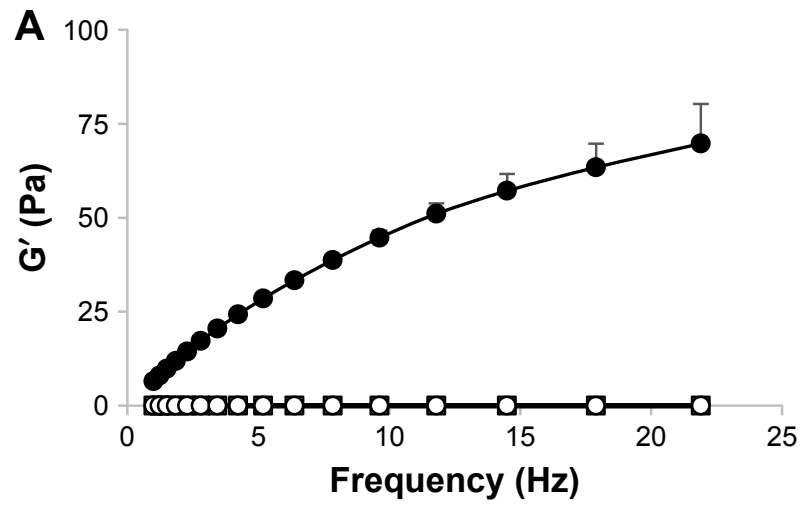

$$
\longrightarrow \text { ALG 0.5\% w/w } \longrightarrow \text { DEX } 30 \% \mathrm{w} / \mathrm{w}
$$$$
\longrightarrow \text { ALG } 0.5 \% \mathrm{w} / \mathrm{w}-\mathrm{DEX} 30 \% \mathrm{w} / \mathrm{w} \text { mixture }
$$

Figure SI (Continued) on polymer molecular weight, chain morphology, and affinity of the solvent used. ${ }^{1}$ In Figure S2, the viscosity parameters $\eta_{0}$ and $\eta_{\infty}$, indexes of DEX solution structure in deionized water at low and high shear rate, respectively, are reported against DEX concentration. Both the viscosity parameters exponentially increase on increasing DEX concentration. In the inset of Figure S2, log-log profiles of the viscosity parameters $\left(\eta_{0}\right.$ and $\left.\eta_{\infty}\right)$ vs DEX concentration allow the identification of DEX CEC, which corresponds to the intersection point between two straight lines that best represent, on a statistical basis, the experimental data. DEX CEC results equal to $12.1 \% \mathrm{w} / \mathrm{w}$. Analogous viscosity parameters $\left(\eta_{0}\right.$ and $\left.\eta_{\infty}\right)$ have been observed for DEX solutions prepared in $\mathrm{pH} 5.5$ phosphate buffer (data not shown), indicating that the presence of salts does not influence the rheological behavior of DEX aqueous solutions and, thus, chain entanglement.

\section{Comparison of the rheological behavior of DEX and PEO aqueous solutions}

Viscosity curves were obtained for DEX and PEO aqueous solutions ( $1 \% \mathrm{w} / \mathrm{w}$ concentration) by means of a rotational rheometer (MCR 102), using a C50-1 cone $(\varnothing=50 \mathrm{~mm}$ and $\vartheta=1^{\circ}$ ) as measuring system. Measurements were performed in a shear rate ranging from 10 to $1,000 \mathrm{~s}^{-1}$ at $33^{\circ} \mathrm{C}$ (temperature to which the polymer solutions were subjected during the electrospinning process).

In Figure S3, the viscosity profiles of DEX and $\mathrm{PEO}$ aqueous solutions prepared at the same concentration $(1 \% \mathrm{w} / \mathrm{w})$ are compared. It can be observed that the two polymer solutions are characterized by a completely different rheological
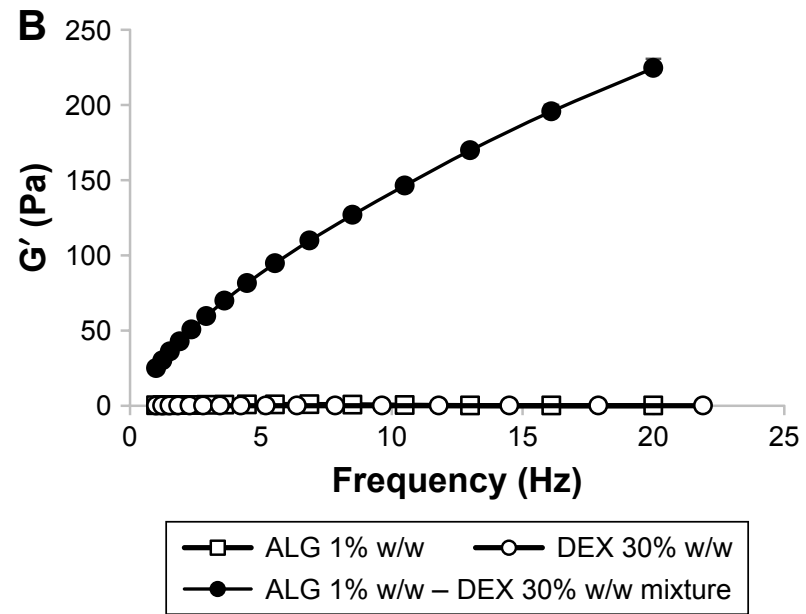


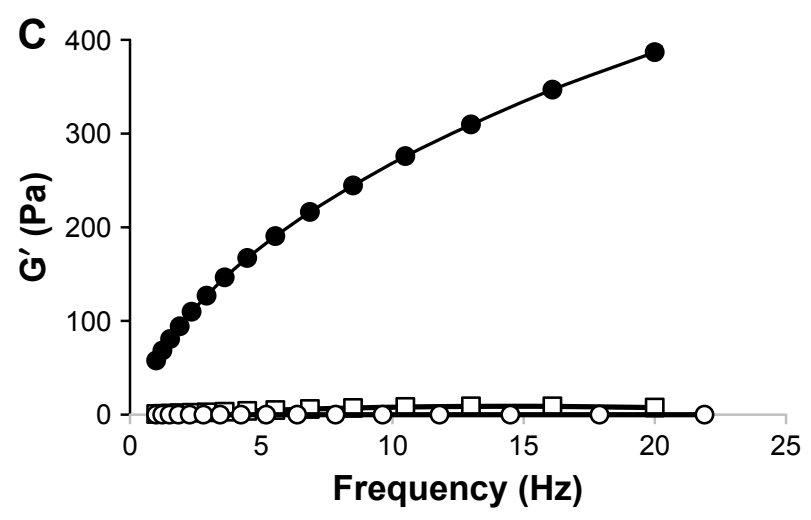

$\longrightarrow$ - ALG $1.5 \% \mathrm{w} / \mathrm{w} \longrightarrow \mathrm{D} \longrightarrow \mathrm{DEX} 30 \% \mathrm{w} / \mathrm{w}$
$\longrightarrow$ ALG $1.5 \% \mathrm{w} / \mathrm{w}-\mathrm{DEX} 30 \% \mathrm{w} / \mathrm{w}$ mixture

Figure SI G' vs frequency profiles of blends of $30 \% w / w$ DEX with different $A L G$ concentrations and of the individual polymer solutions (mean values \pm SD; $n=3$ ); (A) ALG $0.5 \% \mathrm{w} / \mathrm{w}-D E X 30 \% \mathrm{w} / \mathrm{w}$, (B) ALG I\% w/w-DEX 30\% w/w, (C) ALG I.5\% w/w-DEX 30\% w/w.

Abbreviations: DEX, dextran; ALG, alginate.

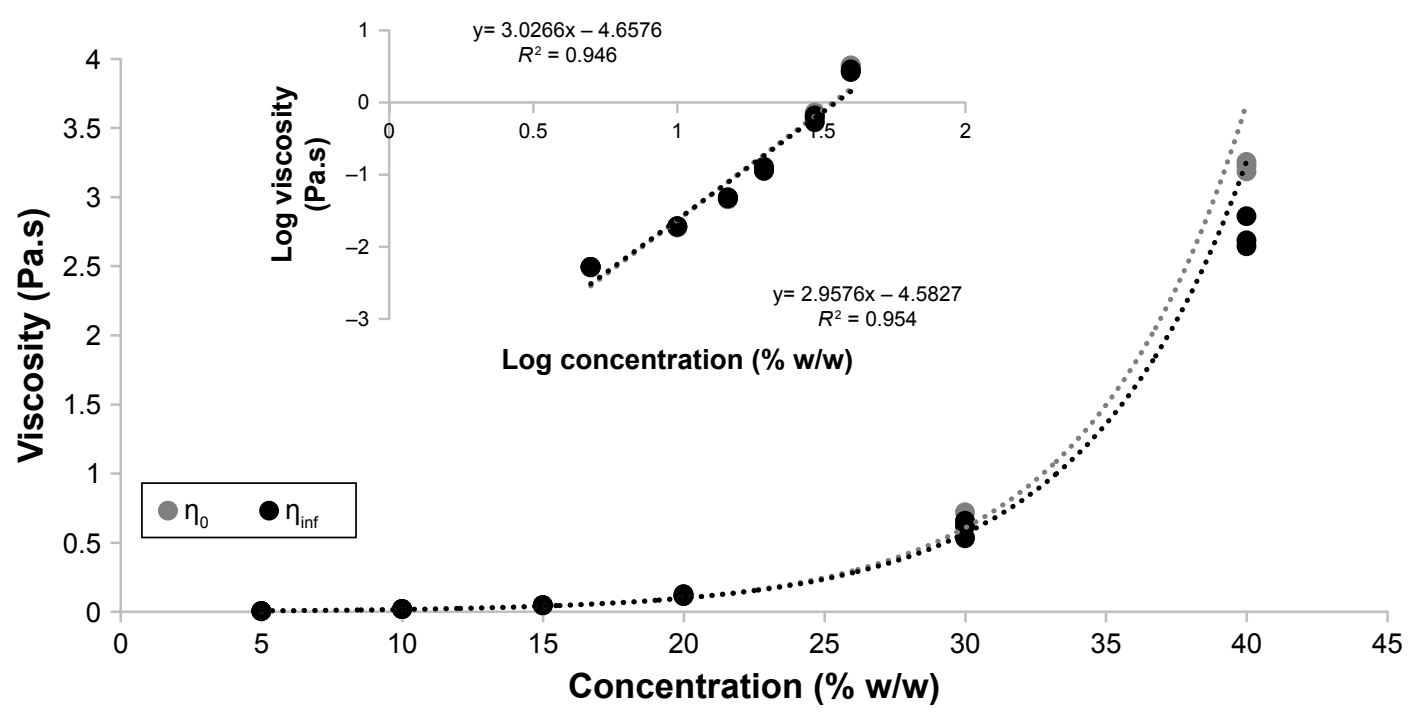

Figure S2 Viscosity parameters $\left(\eta_{0}\right.$ and $\left.\eta_{\infty}\right)$ as a function of dextran concentration for solutions prepared in distilled water.

Note: Log-log profiles are reported in the inset.

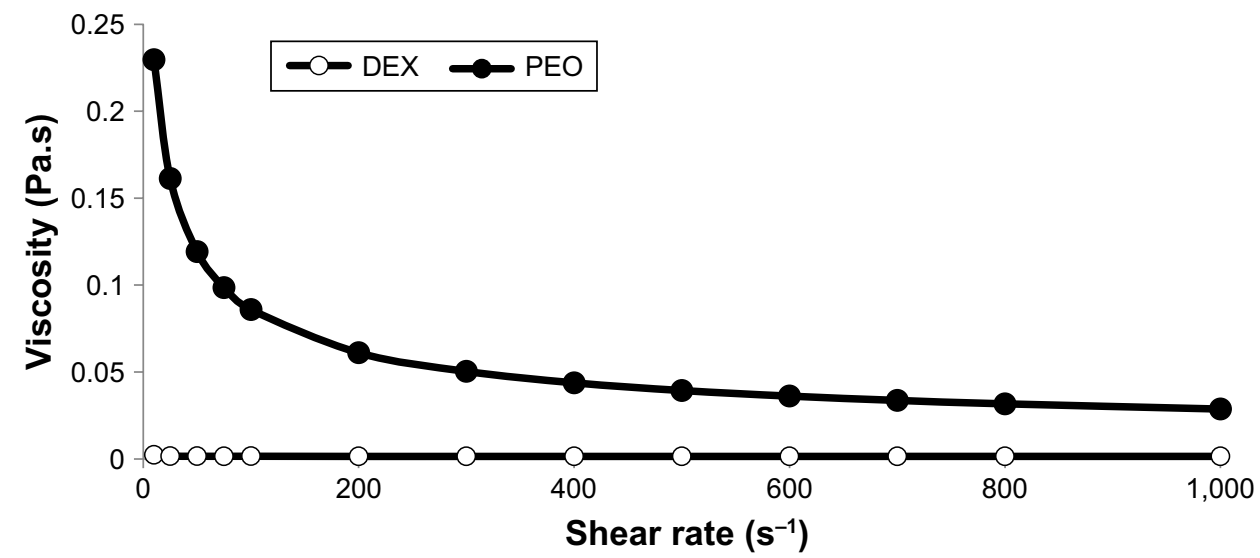

Figure S3 Comparison of viscosity profiles, observed at $33^{\circ} \mathrm{C}$, of DEX and PEO solutions, both prepared in deionized water at the concentration of I\% w/w (mean values $\pm S D ; n=3$ ).

Abbreviations: DEX, dextran; PEO, polyethylene oxide. 
behavior: while PEO solution shows a pseudoplastic behavior, indicated by a decrease of viscosity on increasing shear rate, DEX solution prepared at the same concentration is characterized by a Newtonian behavior (ie, a viscosity independent from shear rate).

\section{Reference}

1. Rossi S, Ferrari F, Bonferoni MC, Caramella C. Characterization of chitosan hydrochloride-mucin rheological interaction: influence of polymer concentration and polymer:mucin weight ratio. Eur J Pharm Sci. 2001;12(4):479-485.

\section{Publish your work in this journal}

The International Journal of Nanomedicine is an international, peerreviewed journal focusing on the application of nanotechnology in diagnostics, therapeutics, and drug delivery systems throughout the biomedical field. This journal is indexed on PubMed Central, MedLine, CAS, SciSearch $\AA$, Current Contents ${ }^{\circledR} /$ Clinical Medicine,
Journal Citation Reports/Science Edition, EMBase, Scopus and the Elsevier Bibliographic databases. The manuscript management system is completely online and includes a very quick and fair peer-review system, which is all easy to use. Visit http://www.dovepress.com/ testimonials.php to read real quotes from published authors. 